\title{
On the Role of Basic Production Terms in an Activator-Inhibitor System Modeling Biological Pattern Formation
}

\author{
By \\ Kanako SuzUKI* and Izumi TAKAGi ${ }^{\dagger}$ \\ (Tohoku University, Japan) \\ Dedicated to the memory of the late Professor Tetsuro Miyakawa
}

\begin{abstract}
Considered is the asymptotic behavior of solutions to a class of reactiondiffusion systems comprised of an activator and an inhibitor, which includes the system proposed by Gierer and Meinhardt as a model of biological pattern formation. By the basic production terms we mean those independent of the unknown functions. We prove that, when the basic production term for the activator is absent, some solutions with large initial data converge to the trivial state, i.e., the activator vanishes identically. Also, we demonstrate that there exist solutions which start from large initial data and converge to a small stationary solution in the case where the basic production term for the inhibitor is nontrivial and that for the activator is sufficiently small.

Key Words and Phrases. Activator-inhibitor system, Pattern formation, Collapse of patterns, Steady-state patterns.

2000 Mathematics Subject Classification Numbers. 35K50, 35K57, 92C15.
\end{abstract}

\section{Introduction and statement of results}

\subsection{Problem}

In the celebrated paper [19], A. M. Turing found that the reaction between two chemicals with different diffusion rates may cause the destabilization of the spatially homogeneous state, thus leading to the formation of nontrivial spatial structure. Developing Turing's idea, A. Gierer and H. Meinhardt [1] proposed several reaction-diffusion systems to model biological pattern formation. Among them is the following system of equations consisting of a slowly diffusing activator and a rapidly diffusing inhibitor:

\footnotetext{
* Supported in part by the Grant-in-Aid for Young Scientists (B) 20740087, MEXT and (B) 23740118, JSPS.

$\dagger$ Supported in part by the Grant-in-Aid for Scientific Research (A) 18204010 and (A) 22244010 , JSPS.
} 
$(\mathrm{GM})$

$$
\begin{cases}a_{t}=D_{a} a_{x x}-\mu a+\rho\left(c \frac{a^{2}}{h}+\rho_{0}\right) & (0<x<l, t>0), \\ h_{t}=D_{h} h_{x x}-v h+c^{\prime} \rho^{\prime} a^{2} & (0<x<l, t>0), \\ a_{x}=h_{x}=0 \quad(x=0, l, t>0), & \end{cases}
$$

where $a=a(x, t)$ and $h=h(x, t)$ denotes the respective concentrations of the activator and the inhibitor; $D_{a}, D_{h}, \mu, v, c, c^{\prime}, \rho_{0}$ are assumed to be positive constants; whereas $\rho(x)$ and $\rho^{\prime}(x)$ are positive functions. They assumed that a change in cells or tissue takes place in the region where the activator concentration is high, and used this system to simulate the regeneration and transplantation experiments on hydra. In their computer simulations the spatial dependence of $\rho$ and $\rho^{\prime}$ reflects the "polarity" which is believed to exist along the body column of hydra to point the direction of the head. This system of equations is called an activator-inhibitor system with different sources, and has been widely used to model various biological pattern formation. A naive question raised immediately is why they introduced the basic production term $\rho \rho_{0}$ only for the activator. The purpose of the present paper is to answer this question.

The biological pattern formation often happens in nonhomogeneous environments and sometimes the spatial inhomogeneity plays an essential rôle in selecting, e.g., the orientation. Therefore, it is natural to study reactiondiffusion systems with coefficients depending on spatial variables.

Let $\Omega$ be a bounded domain in $\boldsymbol{R}^{N}$ with smooth boundary $\partial \Omega$. Suppose $\left(d_{i, j}^{(a)}(x)\right)$ and $\left(d_{i, j}^{(h)}(x)\right)$ are $N \times N$ real symmetric matrices for which there exist positive constants $d_{a}$ and $d_{h}$ such that

$$
|\xi|^{2} \leq \sum_{i, j=1}^{N} d_{i, j}^{(a)} \xi_{i} \xi_{j} \leq d_{a}|\xi|^{2}, \quad|\xi|^{2} \leq \sum_{i, j=1}^{N} d_{i, j}^{(h)} \xi_{i} \xi_{j} \leq d_{h}|\xi|^{2}
$$

for all $\xi \in \boldsymbol{R}^{N}$ and $x \in \Omega$. We assume that the function $d_{i, j}^{(a)}(x)$ and $d_{i, j}^{(h)}(x)$ and their first order derivatives $\nabla d_{i, j}^{(a)}(x), \nabla d_{i, j}^{(h)}(x)$ are Hölder continuous in $\bar{\Omega}$. We define two elliptic partial differential operators $\boldsymbol{\Lambda}_{a}$ and $\boldsymbol{\Lambda}_{h}$ by

$$
\boldsymbol{\Lambda}_{a}=\sum_{i, j=1}^{N} \frac{\partial}{\partial x_{i}}\left(d_{i, j}^{(a)}(x) \frac{\partial}{\partial x_{j}}\right), \quad \boldsymbol{\Lambda}_{h}=\sum_{i, j=1}^{N} \frac{\partial}{\partial x_{i}}\left(d_{i, j}^{(h)}(x) \frac{\partial}{\partial x_{j}}\right)
$$

and associated boundary operators $\boldsymbol{B}_{a}$ and $\boldsymbol{B}_{h}$ by

$$
\boldsymbol{B}_{a}=\sum_{i, j=1}^{N} v_{i} d_{i, j}^{(a)} \frac{\partial}{\partial x_{j}}, \quad \boldsymbol{B}_{h}=\sum_{i, j=1}^{N} v_{i} d_{i, j}^{(h)} \frac{\partial}{\partial x_{j}},
$$


where $v=\left(v_{1}, \ldots, v_{N}\right)$ denotes the unit outer normal vector to $\partial \Omega$. The following is a slightly generalized form of the activator-inhibitor system proposed by Gierer and Meinhardt:

$$
\left\{\begin{array}{l}
\frac{\partial A}{\partial t}=\varepsilon^{2} \boldsymbol{\Lambda}_{a} A-\mu_{a}(x) A+\rho_{a}(A, H, x) \frac{A^{p}}{H^{q}}+\sigma_{a}(x) \text { for } x \in \Omega, t>0, \\
\tau \frac{\partial H}{\partial t}=D \boldsymbol{\Lambda}_{h} H-\mu_{h}(x) H+\rho_{h}(A, H, x) \frac{A^{r}}{H^{s}}+\sigma_{h}(x) \text { for } x \in \Omega, t>0 .
\end{array}\right.
$$

We impose the non-flux boundary condition and the initial condition

$$
\begin{array}{ll}
\boldsymbol{B}_{a} A=0 \quad \text { and } & \boldsymbol{B}_{h} H=0 \quad \text { for } x \in \partial \Omega, t>0, \\
A(x, 0)=A_{0}(x), & H(x, 0)=H_{0}(x) \quad \text { for } x \in \Omega .
\end{array}
$$

Here $\varepsilon, D$ and $\tau$ are positive constants; the removal rates $\mu_{a}(x), \mu_{h}(x)$ are Hölder continuous functions on $\bar{\Omega}$ such that

$$
0<k_{1}^{(a)} \leq \mu_{a}(x) \leq k_{2}^{(a)}, \quad 0<k_{1}^{(h)} \leq \mu_{h}(x) \leq k_{2}^{(h)} \quad \text { for all } x \in \bar{\Omega} ;
$$

the cross-reaction coefficients $\rho_{a}(A, H, x)$ and $\rho_{h}(A, H, x)$ are positive functions defined for $-\infty<A<+\infty,-\infty<H<+\infty, x \in \bar{\Omega}$ which are continuously differentiable in $(A, H)$ and Hölder continuous in $x \in \bar{\Omega}$. Moreover, there are positive constants $c_{a}, C_{a}, c_{h}, C_{h}$ such that

$$
\begin{array}{ll}
0<c_{a}<\rho_{a}(A, H, x) \leq C_{a}, & \left|\frac{\partial \rho_{a}}{\partial A}(A, H, x)\right|,\left|\frac{\partial \rho_{a}}{\partial H}(A, H, x)\right| \leq C_{a}, \\
0<c_{h} \leq \rho_{h}(A, H, x) \leq C_{h}, & \left|\frac{\partial \rho_{h}}{\partial A}(A, H, x)\right|,\left|\frac{\partial \rho_{h}}{\partial H}(A, H, x)\right| \leq C_{h}
\end{array}
$$

for $A \geq 0, H \geq 0, x \in \bar{\Omega}$. Concerning the basic production terms $\sigma_{a}$ and $\sigma_{h}$, we assume

$$
\sigma_{a}, \sigma_{h} \in C^{\gamma}(\bar{\Omega}), \quad \text { and } \quad \sigma_{a}(x) \geq 0, \sigma_{h}(x) \geq 0 \text { on } \bar{\Omega}
$$

and as to the initial data, we assume

$$
\begin{aligned}
& A_{0}, H_{0} \in C^{2+\gamma}(\bar{\Omega}),\left.\quad \boldsymbol{B}_{a} A_{0}\right|_{\partial \Omega}=\left.\boldsymbol{B}_{h} H_{0}\right|_{\partial \Omega}=0 \quad \text { and } \\
& A_{0}(x)>0, H_{0}(x)>0 \text { on } \bar{\Omega},
\end{aligned}
$$

where $0<\gamma<1$. Furthermore, the exponents $p>0, q>0, r>0, s \geq 0$ satisfy

$$
0<\frac{p-1}{r}<\frac{q}{s+1} .
$$

We remark here that we follow [3] for the names of the coefficients $\mu_{a}, \mu_{h}, \rho_{a}, \rho_{h}$. 


\subsection{Quick review of known results on the existence and boundedness of solutions}

From a mathematical point of view, one of the fundamental questions is whether the initial-boundary value problem $(1.1)-(1.3)$ has a solution for all $t>0$ or not. When $\boldsymbol{\Lambda}_{a}$ and $\boldsymbol{\Lambda}_{h}$ are the Laplace operator $\Delta=\sum_{j=1}^{N} \partial^{2} / \partial x_{j}^{2}$ and $\mu_{a}, \mu_{h}$ are constants, there have appeared several results on this question (see, e.g., [14], [7], [21], [4], [2]). In particular, under the assumption that $\min _{x \in \bar{\Omega}} \sigma_{a}(x)>0, \sigma_{h}(x) \equiv 0$ and $(p-1) / r<2 /(N+2)$, Masuda and Takahashi [7] proved not only that the solution exists for all $t>0$ but also that, as $t \rightarrow+\infty$, the set $\left\{(A(x, t), H(x, t)) \in \boldsymbol{R}^{2} \mid x \in \Omega\right\}$ is confined in a fixed rectangle which is independent of the initial data. Meanwhile, Li, Chen and Qin [4] assumed $p-1<r$ and $\min _{x \in \bar{\Omega}} \sigma_{a}(x)>0$ to prove that the solution exists for all $t>0$ in the case where $\rho_{a}(A, H, x) \equiv \rho_{h}(A, H, x) \equiv 1$ and $\rho_{h}(x) \equiv 0$. Jiang [2] and the authors independently obtained similar results on the existence and boundedness of solutions including the case where $\min _{x \in \bar{\Omega}} \sigma_{a}(x)=0$. The method of proving these results carries over the case of nonconstant coefficients without additional difficulties. Putting the known results together, we can state the following Theorems A-C which complement the results by [7], [4], [2] and [15], and give us a complete understanding of the existence and the boundedness of solutions to the generalized system (1.1)-(1.3) in the case $p-1<r$.

Theorem A. Assume, in addition to (1.9), that

$$
p-1<r
$$

and, in addition to (1.7), that

$$
\max _{x \in \bar{\Omega}} \sigma_{a}(x)>0
$$

Then the initial-boundary value problem (1.1)-(1.3) has a unique solution for all $t>0$. Moreover, there exist positive constants $r_{a}, r_{h}, R_{a}, R_{h}$, independent of the initial data $\left(A_{0}(x), H_{0}(x)\right)$, such that

$$
\begin{aligned}
& r_{a} \leq \liminf _{t \rightarrow+\infty} \min _{x \in \bar{\Omega}} A(x, t) \leq \limsup _{t \rightarrow+\infty} \max _{x \in \bar{\Omega}} A(x, t) \leq R_{a}, \\
& r_{h} \leq \liminf _{t \rightarrow+\infty} \min _{x \in \bar{\Omega}} H(x, t) \leq \limsup _{t \rightarrow+\infty} \max _{x \in \bar{\Omega}} H(x, t) \leq R_{h} .
\end{aligned}
$$

Theorem B. Assume that (1.10) is satisfied in addition to (1.9). Suppose that

$$
\sigma_{a}(x) \equiv 0 \quad \text { and } \quad \max _{x \in \bar{\Omega}} \sigma_{h}(x)>0
$$


Then the initial-boundary value problem (1.1)-(1.3) has a unique solution for all $t>0$. Moreover, there exist positive constants $r_{h}, R_{a}, R_{h}$ which are independent of the initial data $\left(A_{0}(x), H_{0}(x)\right)$ such that

and

$$
e^{-k_{2}^{(a)} t} \min _{x \in \bar{\Omega}} A_{0}(x) \leq A(x, t) \text { for all } x \in \bar{\Omega}, t>0,
$$

$$
\begin{gathered}
\limsup _{t \rightarrow+\infty} \max _{x \in \bar{\Omega}} A(x, t) \leq R_{a} \\
r_{h} \leq \liminf _{t \rightarrow+\infty} \min _{x \in \bar{\Omega}} H(x, t) \leq \limsup _{t \rightarrow+\infty} \max _{x \in \bar{\Omega}} H(x, t) \leq R_{h} .
\end{gathered}
$$

Theorem C. Assume that (1.10) is satisfied in addition to (1.9). Suppose that

$$
\sigma_{a}(x) \equiv 0 \quad \text { and } \quad \sigma_{h}(x) \equiv 0 .
$$

Then the initial-boundary value problem (1.1)-(1.3) has a unique solution for all $t>0$. Moreover, there are positive constants $\lambda$ and $\mu$ which are dependent only on $p, q, r, s, \tau, k_{1}^{(a)}, k_{2}^{(h)}, C_{a}$ and $c_{h}$, and a positive constant $C$ depending on initial data $\left(A_{0}(x), H_{0}(x)\right)$ such that

$$
e^{-k_{2}^{(a)} t} \min _{x \in \bar{\Omega}} A_{0}(x) \leq A(x, t) \leq C e^{\lambda t}, \quad e^{-k_{2}^{(h)} t / \tau} \min _{x \in \bar{\Omega}} H_{0}(x) \leq H(x, t) \leq C e^{\mu t}
$$

for all $t>0$ and $x \in \bar{\Omega}$.

Some remarks on Theorems $\mathrm{A}-\mathrm{C}$ are in order. First, all the three theorems assume that $p-1<r$, which is important to rule out the occurrence of finite time blow-up of solutions. Indeed, [4] and [8] showed in the case of a homogeneous medium that if $p-1>r$, then there exist solutions of (1.1)-(1.3) with $\sigma_{a}(x) \equiv \sigma_{h}(x) \equiv 0$ which blow up in finite time. Here and in what follows by the a homogeneous medium we mean that $\boldsymbol{\Lambda}_{a}=\boldsymbol{\Lambda}_{h}=\Delta$, $\mu_{a} \equiv \mu_{h} \equiv \rho_{a} \equiv \rho_{h} \equiv 1$, and both of $\sigma_{a}$ and $\sigma_{h}$ are nonnegative constants.

Second, in [8] it is proved in the case of a homogeneous medium that if $p-1 \leq r, q \geq s+1$ and $s+1<(p-1) \tau \leq q$, then some solutions of (1.1)-(1.3) with $\sigma_{a}(x) \equiv \sigma_{h}(x) \equiv 0$ exist for all $t>0$, but they are unbounded. By virtue of Theorem A, all solutions are bounded if $\sigma_{a}$ is nontrivial.

\subsection{Main results}

As is seen from the known results stated in the previous subsection, if $\sigma_{a}(x) \not \equiv 0$ then we are in a nice situation in the sense that all the solutions of the initial-boundary value problem (1.1)-(1.3) exist for all $t>0$ and will be confined in a fixed rectangle eventually, provided that $p-1<r$. If $\sigma_{a}(x) \equiv 0$ and $p-1<r$, then solutions still exist for all $t>0$ but may become unbounded when $\sigma_{h}(x) \equiv 0$. This may be a partial answer to the naive question raised 
in the introduction. However, in this paper we report a new phenomenon, called the collapse of patterns, which we suspect is another reason for adding the basic production term only to the activator. In some numerical simulations, we encounter a solution starting from an almost uniform initial value that develops localization in the activator concentration for a while, but it begins to oscillate and eventually converges uniformly to the trivial state $A \equiv 0$. Indeed, for the homogeneous medium with $\sigma_{a}(x) \equiv \sigma_{h}(x) \equiv 0, \mathrm{Wu}$ and $\mathrm{Li}$ [21] found that there are solutions of (1.1)-(1.3) with large initial data such that $(A(x, t), H(x, t)) \rightarrow(0,0)$ uniformly on $\bar{\Omega}$ as $t \rightarrow+\infty$. We call vaguely this phenomenon the collapse of patterns. As a matter of fact, Yanagihara [22] proved the existence of solutions which converge to $(0,0)$ for $(\mathrm{GM})$ with $\rho_{0}=0$ as early as in the late 1970 's, assuming that the initial data is sufficiently small. One of the purposes of the present paper is to study this phenomenon in detail and know the structure of kinetics which leads to the collapse of patterns.

For lack of uniform lower bounds for the activator in the case $\sigma_{a}(x) \equiv 0$, Theorems B and C suggest that patterns in the activator can collapse even though $\sigma_{h}(x)>0$. We prove that this is indeed the case. To begin with we introduce two functions $\Sigma_{a, \varepsilon}(x)$ and $\Sigma_{h, D}(x)$ which are respective solutions of the following linear boundary value problems:

$$
\left\{\begin{array}{l}
\varepsilon^{2} \boldsymbol{\Lambda}_{a} \Sigma_{a, \varepsilon}-\mu_{a}(x) \Sigma_{a, \varepsilon}+\sigma_{a}(x)=0 \text { for } x \in \Omega, \\
\boldsymbol{B}_{a} \Sigma_{a, \varepsilon}=0 \text { for } x \in \partial \Omega
\end{array}\right.
$$

and

$$
\left\{\begin{array}{l}
D \Lambda_{h} \Sigma_{h, D}-\mu_{h}(x) \Sigma_{h, D}+\sigma_{h}(x)=0 \text { for } x \in \Omega, \\
\boldsymbol{B}_{h} \Sigma_{h, D}=0 \text { for } x \in \partial \Omega
\end{array}\right.
$$

By the standard theory of linear elliptic partial differential operators, each of these boundary value problems has a unique classical solution. The solution $\Sigma_{a, \varepsilon}$ of (1.11) is strictly positive whenever $\max _{x \in \bar{\Omega}} \sigma_{a}(x)>0$, while $\Sigma_{a, \varepsilon}(x) \equiv 0$ if $\sigma_{a}(x) \equiv 0$. The same is true for the solution $\Sigma_{h, D}$ of (1.12).

It is convenient to classify the basic production terms as

Case I: $\quad \sigma_{a}(x) \equiv 0$ and $\sigma_{h}(x) \equiv 0 ; \quad$ Case II: $\quad \sigma_{a}(x) \equiv 0$ and $\sigma_{h}(x) \not \equiv 0$;

Case III: $\quad \sigma_{a}(x) \not \equiv 0$ and $\sigma_{h}(x) \not \equiv 0 ; \quad$ Case IV: $\quad \sigma_{a}(x) \not \equiv 0$ and $\sigma_{h}(x) \equiv 0$.

The following theorem slightly generalizes the result by $\mathrm{Wu}$ and $\mathrm{Li}[21]$ to inhomogeneous media.

Theorem 1.1 (Final phase of collapse of patterns, Cases I and II). Let $\sigma_{a}(x) \equiv 0$. Assume that $\tau$ satisfies $\tau>q k_{2}^{(h)} /(p-1) k_{1}^{(a)}$ and the initial data satisfy

$$
\left(\min _{x \in \bar{\Omega}} H_{0}(x)\right)^{q}>\frac{C_{a}(p-1)}{k_{1}^{(a)}(p-1)-q k_{2}^{(h)} / \tau}\left(\max _{x \in \bar{\Omega}} A_{0}(x)\right)^{p-1} .
$$


Then the solution $(A(x, t), H(x, t))$ of $(1.1)-(1.3)$ satisfies

$$
0<\max _{x \in \bar{\Omega}} A(x, t) \leq C e^{-k_{1}^{(a)} t}, \quad \max _{x \in \bar{\Omega}}\left|H(x, t)-\Sigma_{h, D}(x)\right| \leq C e^{-k_{1}^{(h)} t / \tau},
$$

in which $C$ is a positive constant depending on $\left(A_{0}(x), H_{0}(x)\right)$, and $\Sigma_{h, D}(x)$ is the solution of (1.12).

We emphasize that Theorem 1.1 is a "semi-global" result in the sense that the initial data $\left(A_{0}(x), H_{0}(x)\right)$ may be taken very far from the limit $\left(0, \Sigma_{h, D}(x)\right)$. In Case II, we can prove the following:

Theorem 1.2 (Final phase of collapse of patterns, Case II). Assume that $\sigma_{a}(x) \equiv 0$ and $\max _{x \in \bar{\Omega}} \sigma_{h}(x)>0$. Let

$$
\delta_{h}=\min _{x \in \bar{\Omega}} \Sigma_{h, D}(x) \quad \text { and } \quad \Gamma_{h}=\max _{x \in \bar{\Omega}} \Sigma_{h, D}(x) .
$$

If the initial data $\left(A_{0}(x), H_{0}(x)\right)$ satisfies

$$
\begin{aligned}
\min & \left\{\left(\left(\delta_{h} / \Gamma_{h}\right)^{k_{2}^{(h)} / k_{1}^{(h)}} \min _{x \in \bar{\Omega}} H_{0}(x)\right)^{q},\left(\delta_{h} k_{1}^{(h)} / k_{2}^{(h)}\right)^{q}\right\} \\
& >\frac{C_{a}}{k_{1}^{(a)}}\left(\max _{x \in \bar{\Omega}} A_{0}(x)\right)^{p-1},
\end{aligned}
$$

then

$$
0<\max _{x \in \bar{\Omega}} A(x, t) \leq C e^{-k_{1}^{(a)} t}, \quad \max _{x \in \bar{\Omega}}\left|H(x, t)-\Sigma_{h, D}(x)\right| \leq C e^{-k_{1}^{(h)} t / \tau} .
$$

Here $C$ is a positive constant depending also on $\left(A_{0}(x), H_{0}(x)\right)$, and $\Sigma_{h, D}(x)$ is a solution of (1.12).

We note that, in Case I, the largeness of $\tau$ is necessary for the collapse of pattern to occur, as the following proposition shows.

Proposition 1.3. Let $\sigma_{a}(x) \equiv \sigma_{h}(x) \equiv 0$. If there exists a solution $(A(x, t), H(x, t))$ of $(1.1)-(1.3)$ satisfying (i) $H(x, t)^{q}>\rho_{a}(A, H, x) A(x, t)^{p-1} / \mu_{a}(x)$ for all $x \in \bar{\Omega}$ and $t>0$ and (ii) $(A(x, t), H(x, t)) \rightarrow(0,0)$ as $t \rightarrow+\infty$, then $\tau \geq q k_{1}^{(h)} /\left(k_{2}^{(a)}(p-1)\right)$.

By Theorem A it is tempting to guess that patterns never collapse as long as $\sigma_{a}(x) \not \equiv 0$. However, in Case III with $\max _{x \in \bar{\Omega}} \sigma_{a}(x)$ being sufficiently small, Theorem 1.5 below gives a stable stationary solution of (1.1)-(1.2) in the neighborhood of $(A, H)=\left(\Sigma_{a, \varepsilon}, \Sigma_{h, D}\right)$ which we call an almost decoupled pattern. This leaves room for the occurrence of collapse in Case III, too. 
First we give a precise definition of an almost decoupled pattern.

Definition 1.4. A stationary solution $(A(x), H(x))$ of $(1.1)-(1.2)$ is called an almost decoupled pattern if

and

$$
-\mu_{a}(x)+\rho_{a}(A(x), H(x), x) \frac{A(x)^{p-1}}{H(x)^{q}}<0
$$

$$
-\mu_{h}(x)+\rho_{h}(A(x), H(x), x) \frac{A(x)^{r}}{H(x)^{s+1}}<0
$$

for all $x \in \bar{\Omega}$.

Note that if $\sigma_{h}(x) \equiv 0$, then there is no almost decoupled pattern, since

$$
0=\int_{\Omega}\left\{D \boldsymbol{\Lambda}_{h} H-\left(\mu_{h}-\rho_{h} \frac{A^{r}}{H^{s+1}}\right) H\right\} d x=\int_{\Omega}-\left(\mu_{h}-\rho_{h} \frac{A^{r}}{H^{s+1}}\right) H d x
$$

We recall here the definition of the stability of stationary solutions. A stationary solution $\left(A_{*}(x), H_{*}(x)\right)$ of $(1.1)-(1.3)$ is said to be stable if for any $\eta>0$ there exists $\delta>0$ such that $\left\|A_{0}-A_{*}\right\|_{W^{2, P}(\Omega)}+\left\|H_{0}-H_{*}\right\|_{W^{2, P}(\Omega)}<\delta$ implies $\left\|A(\cdot, t)-A_{*}\right\|_{W^{2, P}(\Omega)}+\left\|H(\cdot, t)-H_{*}\right\|_{W^{2, P}(\Omega)}<\eta$ for all $t>0$, in which $P>N$. Here, $W^{2, P}(\Omega)$, with $P>N$, denotes the Banach space consisting of all functions in $L^{P}(\Omega)$ whose partial derivatives of order up to two are also in $L^{P}(\Omega)$. We say that a stationary solution $\left(A_{*}(x), H_{*}(x)\right)$ is asymptotically stable if it is stable and moreover $\left\|A(\cdot, t)-A_{*}\right\|_{L^{\infty}(\Omega)}+\left\|H(\cdot, t)-H_{*}\right\|_{L^{\infty}(\Omega)} \rightarrow 0$ as $t \rightarrow+\infty$. Note that if it is asymptotically stable then we actually have $\left\|A(\cdot, t)-A_{*}\right\|_{W^{2, P}(\Omega)}+\left\|H(\cdot, t)-H_{*}\right\|_{W^{2, P}(\Omega)} \rightarrow 0$ as $t \rightarrow+\infty$ by the standard boot-strap method. Finally, a stationary solution $\left(A_{*}, H_{*}\right)$ is said to be unstable if it is not stable.

Theorem 1.5 (Almost decoupled pattern). Let $\max _{x \in \bar{\Omega}} \sigma_{a}(x)>0$. Moreover, assume that $\min _{x \in \bar{\Omega}} \sigma_{a}(x) \geq \gamma_{a}\left(\max _{x \in \bar{\Omega}} \sigma_{a}(x)\right)^{p}$ if $0<r<1$, where $\gamma_{a}$ is a positive constant independent of $\sigma_{a}(x)$. Let $\max _{x \in \bar{\Omega}} \sigma_{h}(x)>0$. Then there exists a positive number $m_{0}$ such that if $\max _{x \in \bar{\Omega}} \sigma_{a}(x) \leq m_{0}$, then (1.1)-(1.2) has a stationary solution $\left(A_{*}(x), H_{*}(x)\right)$ which satisfies

$$
\begin{aligned}
& \left\|A_{*}-\Sigma_{a, \varepsilon}\right\|_{L^{\infty}} \leq C\left(\max _{x \in \bar{\Omega}} \sigma_{a}(x)\right)^{p}, \\
& \left\|H_{*}-\Sigma_{h, D}\right\|_{L^{\infty}} \leq C\left(\max _{x \in \bar{\Omega}} \sigma_{a}(x)\right)^{r},
\end{aligned}
$$

where $C$ is a positive constant and $\Sigma_{a, \varepsilon}, \Sigma_{h, D}$ are solutions of (1.11), (1.12), respectively. Furthermore, this stationary solution is asymptotically stable. 
Remark 1.6. (1) The positive constant $\gamma_{a}$ will be specified in the paragraph containing (3.20) in Section 3.1.

(2) The constant $m_{0}$ depends on $\mu_{a}, \rho_{a}, D, \boldsymbol{\Lambda}_{h}, \mu_{h}, \rho_{h}, \sigma_{h}$ and $(p, q, r, s)$, but independent of $\varepsilon, \Lambda_{a}$ and $\tau$. (See the proof of Theorem 1.5 in Section 3.) In Proposition 3.5 we shall prove that actually the solution converges exponentially as $t \rightarrow+\infty$.

We now introduce important quantities $\kappa_{a}$ and $K_{a}$ to state the result in Case III. Let $0<\kappa_{a}<K_{a}$ be two positive roots of the algebraic equation

$$
-k_{1}^{(a)} \xi+\frac{C_{a}}{\delta_{h}^{q}} \xi^{p}+\max _{x \in \bar{\Omega}} \sigma_{a}(x)=0,
$$

where $\delta_{h}$ is defined in (1.14), $\max _{x \in \bar{\Omega}} \sigma_{a}(x) \leq m_{0}$ and $m_{0}$ is the positive constant appearing in Theorem 1.5. It is not difficult to check that, as $\max _{x \in \bar{\Omega}} \sigma_{a}(x) \rightarrow 0$,

$$
\begin{aligned}
\kappa_{a} & =\frac{1}{k_{1}^{(a)}} \max _{x \in \bar{\Omega}} \sigma_{a}(x)+O\left(\left(\max _{x \in \bar{\Omega}} \sigma_{a}(x)\right)^{p}\right) \\
K_{a} & =\left(\frac{k_{1}^{(a)} \delta_{h}^{q}}{C_{a}}\right)^{1 /(p-1)}-\frac{k_{1}^{(a)}}{p-1} \max _{x \in \bar{\Omega}} \sigma_{a}(x)+o\left(\max _{x \in \bar{\Omega}} \sigma_{a}(x)\right) .
\end{aligned}
$$

Theorem 1.7 (Collapse to almost decoupled pattern, Case III). Under the same assumption as in Theorem 1.5, if the initial data $\left(A_{0}(x), H_{0}(x)\right)$ satisfies

$$
\max _{x \in \bar{\Omega}} A_{0}(x)<K_{a}, \quad H_{0}(x) \geq \max _{x \in \bar{\Omega}} \Sigma_{h, D}(x),
$$

then

$$
\max _{x \in \bar{\Omega}}\left(\left|A(x, t)-A_{*}(x)\right|+\left|H(x, t)-H_{*}(x)\right|\right) \leq C e^{-\gamma t} \quad \text { for all } t \geq 0 .
$$

Here $\left(A_{*}, H_{*}\right)$ is the almost decoupled pattern given by Theorem 1.5, and $C$ and $\gamma$ are positive constants depending also on $\left(A_{0}, H_{0}\right)$.

Remark 1.8. (1) Proposition 1.3 gives a necessary condition on $\tau$ for the existence of a solution $(A(x, t), H(x, t))$ of $(1.1)-(1.3)$ converging to $(0,0)$ as $t \rightarrow+\infty$ in Case I. Since $k_{1}^{(h)} / k_{2}^{(h)} \leq 1 \leq k_{2}^{(a)} / k_{1}^{(a)}$, the sufficient condition on $\tau$ imposed in Theorem 1.1 is stronger than the necessary condition.

(2) We notice that in Case III we can apply both Theorems 1.1 and 1.2. If $\tau$ is large and $\min _{x \in \bar{\Omega}} H_{0}(x)$ is also large, then the sufficient condition given by Theorem 1.1 is weaker. 
Remark 1.9. It is to be noted that in contrast to Theorems A-C, we do not assume any further condition on the exponents other than (1.9) in Theorems 1.1, 1.2 and 1.7, yet we have a bounded solution for all $t>0$ by restricting initial data.

Here we would like to summarize the results on the collapse of patterns. Theorem 1.1 shows that if $\sigma_{a}(x) \equiv \sigma_{h}(x) \equiv 0$, then there is a solution $(A(x, t), H(x, t))$ of $(1.1)-(1.3)$ tending to $(0,0)$ uniformly on $\bar{\Omega}$ provided that $\tau$ is sufficiently large. On the other hand, by Theorem 1.2, the collapse of patterns always occurs when $\max _{x \in \bar{\Omega}} \sigma_{h}(x)>0$ under a condition on the initial data which is independent of the magnitude of $\tau$. In Case IV where $\sigma_{a}(x) \not \equiv 0$ and $\sigma_{h}(x) \equiv 0$, the collapse of patterns never occurs because $A(x, t)$ cannot approach 0 by virtue of Theorem A and there does not exist any almost decoupled pattern due to (1.16). Therefore, we may conclude that the simplest way to avoid the collapse of patterns is to take $\sigma_{a}(x) \not \equiv 0$ and $\sigma_{h}(x) \equiv 0$. For more on the role of basic production terms, see also Concluding Remarks at the end of the paper.

The organization of this paper is as follows. In Section 2, we give a proof of Theorem 1.1 and then we proceed to prove Theorem 1.2 and Proposition 1.3. In Section 3, we prove Theorem 1.5 on the existence and stability of almost decoupled patterns, followed by the proof of Theorem 1.7 which is on the collapse to almost decoupled patterns.

\section{Collapse of patterns}

In this section we will sketch the proofs of Theorems 1.1 and 1.2, and Proposition 1.3.

\subsection{Proof of Theorem 1.1}

We follow the approach due to [21]. See also [16] where we gave a proof for a homogeneous media.

Step 1: Lower bounds

First, we estimate $A(x, t)$ and $H(x, t)$ from below. The following lemma can be proved easily by using the maximum principle.

Lemma 2.1. The solution $(A(x, t), H(x, t))$ of $(1.1)-(1.3)$ with $\sigma_{a}(x) \equiv 0$ satisfies

$$
A(x, t) \geq e^{-k_{2}^{(a)} t} \min _{x \in \bar{\Omega}} A_{0}(x), \quad H(x, t) \geq e^{-k_{2}^{(h)} t / \tau} \min _{x \in \bar{\Omega}} H_{0}(x)
$$

for all $t>0, x \in \Omega$. 
We put $\underline{v}(t)=e^{-k_{2}^{(h)} t / \tau} \min _{x \in \bar{\Omega}} H_{0}(x)$.

Step 2: Upper bounds

In order to derive an estimate of $A(x, t)$ from above, we need the following lemma.

Lemma 2.2. Let $\tau$ satisfy $\tau>q k_{2}^{(h)} /\left((p-1) k_{1}^{(a)}\right)$ and $m_{1}$ be a positive number satisfying

$$
m_{1}^{p-1}<\frac{1}{C_{a}}\left(k_{1}^{(a)}-\frac{q k_{2}^{(h)}}{(p-1) \tau}\right) \underline{v}(0)^{q} .
$$

Then the initial value problem

$$
\frac{d m}{d t}=-k_{1}^{(a)} m+C_{a} \frac{m^{p}}{\underline{v}^{q}}, \quad m(0)=m_{1}
$$

has a unique solution for all $t>0$ and there exists a positive constant $C$ depending on $m_{1}$ and $\underline{v}(0)$ such that $m(t) \leq C e^{-k_{1}^{(a)} t}$.

Note that $C_{a}$ is the constant which appeared in (1.5).

Proof. Put $M(t)=1 / m(t)^{p-1}$. Differentiating $M(t)$ in $t$, we have

$$
\frac{d M}{d t}=(p-1) k_{1}^{(a)} M-(p-1) C_{a} \frac{1}{\underline{v}^{q}} .
$$

Since $M(0)=1 / m_{1}^{p-1}$, we obtain that

$$
M(t)=\frac{1}{m_{1}^{p-1}} e^{k_{1}^{(a)}(p-1) t}-(p-1) C_{a} \int_{0}^{t} \frac{e^{k_{1}^{(a)}(p-1)(t-\xi)}}{\underline{v}(\xi)^{q}} d \xi .
$$

It follows from the definition of $\underline{v}(t)$ that

$$
\begin{aligned}
M(t) & =\frac{1}{m_{1}^{p-1}} e^{k_{1}^{(a)}(p-1) t}-C_{a} \frac{p-1}{\underline{v}(0)^{q}} \int_{0}^{t} e^{k_{1}^{(a)}(p-1)(t-\xi)+q k_{2}^{(h)} \xi / \tau} d \xi \\
& =\left\{\frac{1}{m_{1}^{p-1}}-C_{a} \frac{p-1}{\underline{v}(0)^{q}} \cdot \frac{1-e^{-\left[k_{1}^{(a)}(p-1)-q k_{2}^{(h)} / \tau\right] t}}{k_{1}^{(a)}(p-1)-q k_{2}^{(h)} / \tau}\right\} e^{k_{1}^{(a)}(p-1) t} .
\end{aligned}
$$

If

$$
\frac{1}{m_{1}^{p-1}}>C_{a} \frac{p-1}{\underline{v}(0)^{q}} \cdot \frac{1}{k_{1}^{(a)}(p-1)-q k_{2}^{(h)} / \tau},
$$

then $M(t)>0$ for all $t>0$. Therefore, $m(t)$ exists for all $t>0$ and there is a positive constant $C$ such that $m(t) \leq C e^{-k_{1}^{(a)} t}$. 
From Lemma 2.2, if $\tau>q k_{2}^{(h)} /\left(k_{1}^{(a)}(p-1)\right)$ and (1.13) is satisfied, then the problem

$$
\frac{d \bar{u}}{d t}=-k_{1}^{(a)} \bar{u}+C_{a} \frac{\bar{u}^{p}}{\underline{v}^{q}}, \quad \bar{u}(0)=\max _{x \in \bar{\Omega}} A_{0}(x)
$$

has a solution $\bar{u}(t)$ for all $t>0$ and $\bar{u}(t) \leq C e^{-k_{1}^{(a)} t}$ with some $C>0$ depending on $\bar{u}(0)$ and $\underline{v}(0)$. Applying the maximum principle, we see that

$$
A(x, t) \leq \bar{u}(t) \leq C e^{-k_{1}^{(a)} t} \quad \text { for all } x \in \bar{\Omega}, t>0 .
$$

Next, we consider the initial value problem

$$
\tau \frac{d \bar{v}}{d t}=-k_{1}^{(h)} \bar{v}+C_{h} \frac{\bar{u}^{r}}{\bar{v}^{s}}+\left\|\sigma_{h}\right\|_{L^{\infty}}, \quad \bar{v}(0)=\max _{x \in \bar{\Omega}} H_{0}(x),
$$

where $C_{h}$ is the constant in (1.5) and we put $\left\|\sigma_{h}\right\|_{L^{\infty}}=\max _{x \in \bar{\Omega}} \sigma_{h}(x)$. Because of (2.5), the right-hand side of the first equation of (2.6) is estimated as follows:

$$
\tau \frac{d \bar{v}}{d t} \leq-k_{1}^{(h)} \bar{v}+C_{h} \frac{C^{r} e^{-r k_{1}^{(a)} t}}{\bar{v}^{s}}+\left\|\sigma_{h}\right\|_{L^{\infty}} .
$$

Multiplying the both sides of (2.7) by $\bar{v}^{s}$ and putting $\bar{v}^{s+1}=W$, we have that

$$
\frac{d W}{d t} \leq-\frac{k_{1}^{(h)}(s+1)}{\tau} W+\frac{\left\|\sigma_{h}\right\|_{L^{\infty}}(s+1)}{\tau} W^{s /(s+1)}+\frac{C_{h}^{\prime}(s+1)}{\tau} e^{-r k_{1}^{(a)} t} .
$$

Here $C_{h}^{\prime}=C_{h} C^{r}$. It follows from (2.8) that $W(t) \leq \kappa$ for all $t>0$, where $\kappa$ is the positive root of the algebraic equation $x-\left\|\sigma_{a}\right\|_{L^{\infty}} x^{s /(s+1)} / k_{1}^{(h)}=0$. For a proof, we refer to Lemma 2.2 in [7]. Therefore, the following lemma holds.

Lemma 2.3. Suppose that $\tau>q k_{2}^{(h)} /\left((p-1) k_{1}^{(a)}\right)$ and (1.13) is satisfied. Then the initial value problem (2.6) has a unique bounded solution $\bar{v}(t)$ for all $t>0$.

The maximum principle implies that $H(x, t) \leq \bar{v}(t)$ for $x \in \bar{\Omega}, t>0$.

Step 3: Proof of Theorem 1.1

It is easy to see from $(2.5)$ that $A(x, t) \rightarrow 0$ uniformly on $\bar{\Omega}$ as $t \rightarrow+\infty$. Hence, it remains to consider the behavior of $H(x, t)$ as $t \rightarrow+\infty$.

Let $\Sigma_{h, D}(x)$ be the unique solution of (1.12). Put $W(x, t)=H(x, t)-$ $\Sigma_{h, D}(x)$. Then it is a solution of the following initial-boundary value problem:

$$
\begin{aligned}
\tau \frac{\partial W}{\partial t} & =D \boldsymbol{\Lambda}_{h} W-\mu_{h}(x) W+\rho_{h}(A, H, x) \frac{A^{r}}{H^{s}} \quad \text { for } x \in \Omega, t>0, \\
\boldsymbol{B}_{h} W & =0 \quad \text { for } x \in \partial \Omega, t>0, \\
W(x, 0) & =H_{0}(x)-\Sigma_{h, D}(x) \quad \text { for } x \in \Omega .
\end{aligned}
$$


We note that by $(2.1)$ and $(2.5)$

$$
\rho_{h}(A, H, x) \frac{A^{r}}{H^{s}} \leq C_{h} \frac{C^{r} e^{-r k_{1}^{(a)} t}}{\left[e^{-k_{2}^{(h)} t / \tau} \min _{x \in \bar{\Omega}} H_{0}(x)\right]^{s}}=C^{\prime} e^{-\left[r k_{1}^{(a)}-s k_{2}^{(h)} / \tau\right] t} .
$$

Put $\omega_{0}=\max _{x \in \bar{\Omega}}\left(H_{0}(x)-\Sigma_{h, D}(x)\right)$ and let $\omega(t)$ be the solution of the initial value problem

$$
\tau \frac{d \omega}{d t}=-k_{1}^{(h)} \omega+C^{\prime} e^{-\left[r k_{1}^{(a)}-s k_{2}^{(h)} / \tau\right] t} \quad \text { for } t>0, \quad \text { and } \quad \omega(0)=\omega_{0} .
$$

Then by the maximum principle, we see that $W(x, t) \leq \omega(t)$ for all $x \in \bar{\Omega}$ and $t>0$. Since

$$
\omega(t)=\left\{\omega_{0}+C^{\prime} \frac{1-e^{-\left[r k_{1}^{(a)}-\left(s k_{2}^{(h)}+k_{1}^{(h)}\right) / \tau\right] t}}{\tau r k_{1}^{(a)}-s k_{2}^{(h)}-k_{1}^{(h)}}\right\} e^{-k_{1}^{(h)} t / \tau},
$$

and since

$$
\tau r k_{1}^{(a)}-\left(s k_{2}^{(h)}+k_{1}^{(h)}\right)>k_{2}^{(h)}[q r /(p-1)-(s+1)]+k_{2}^{(h)}-k_{1}^{(h)}>0
$$

by virtue of $\tau>q k_{2}^{(h)} /\left[(p-1) k_{1}^{(a)}\right]$ and (1.9), we have the desired estimate.

\subsection{Proof of Theorem 1.2}

First, we derive a lower bound of $H(x, t)$. By the maximum principle, $H(x, t) \geq H_{1}(x, t)+H_{2}(x, t)$ for all $x \in \bar{\Omega}, t>0$, where $H_{1}$ and $H_{2}$ are unique solutions of

$$
\left\{\begin{array}{l}
\tau \frac{\partial H_{1}}{\partial t}=D \boldsymbol{\Lambda}_{h} H_{1}-\mu_{h}(x) H_{1} \text { for } x \in \Omega, t>0 \\
\boldsymbol{B}_{h} H_{1}=0 \text { for } x \in \partial \Omega, t>0 \\
H_{1}(x, 0)=H_{0}(x) \text { for } x \in \Omega
\end{array}\right.
$$

and

$$
\left\{\begin{array}{l}
\tau \frac{\partial H_{2}}{\partial t}=D \Lambda_{h} H_{2}-\mu_{h}(x) H_{2}+\sigma_{h}(x) \text { for } x \in \Omega, t>0 \\
\boldsymbol{B}_{h} H_{2}=0 \text { for } x \in \partial \Omega, t>0 \\
H_{2}(x, 0)=0 \text { for } x \in \Omega .
\end{array}\right.
$$

By the maximum principle again, we see easily

$$
H_{1}(x, t) \geq e^{-k_{2}^{(h)} t / \tau} \min _{x \in \bar{\Omega}} H_{0}(x), \quad H_{2}(x, t) \geq \max \left\{0, \delta_{h}-\Gamma_{h} e^{-k_{1}^{(h)} t / \tau}\right\},
$$


where $\delta_{h}$ and $\Gamma_{h}$ were defined in (1.14). Put $h_{m}=\min _{x \in \bar{\Omega}} H_{0}(x)$. Since $\delta_{h}-\Gamma_{h} e^{-k_{1}^{(h)} t / \tau}<0$ if $t<t_{0}=\left(\tau \log \left(\Gamma_{h} / \delta_{h}\right)\right) / k_{1}^{(h)}$ and $\delta_{h}-\Gamma_{h} e^{-k_{1}^{(h)} t / \tau}=0$ when $t=t_{0}$, we see that

$$
H(x, t) \geq\left\{\begin{array}{l}
h_{m} e^{-k_{2}^{(h)} t_{0} / \tau} \quad \text { for } 0<t<t_{0}, \\
h_{m} e^{-k_{2}^{(h)} t / \tau}+\delta_{h}-\Gamma_{h} e^{-k_{1}^{(h)} t / \tau} \quad \text { for } t \geq t_{0}
\end{array}\right.
$$

for all $x \in \bar{\Omega}$. We consider the case where $t \geq t_{0}$. Letting

$$
f(\zeta)=h_{m} e^{-k_{2}^{(h)}\left(t_{0}+\zeta\right) / \tau}+\delta_{h}-\Gamma_{h} e^{-k_{1}^{(h)}\left(t_{0}+\zeta\right) / \tau} \quad \text { for } \zeta \geq 0,
$$

we obtain the following: if $h_{m}\left(\delta_{h} / \Gamma_{h}\right)^{k_{2}^{(h)} / k_{1}^{(h)}} \leq \delta_{h} k_{1}^{(h)} / k_{2}^{(h)}$, then $f(\zeta)$ increases monotonically for $\zeta>0$; if $h_{m}\left(\delta_{h} / \Gamma_{h}\right)^{k_{2}^{(h)} / k_{1}^{(h)}}>\delta_{h} k_{1}^{(h)} / k_{2}^{(h)}$, then $f(\zeta)$ has the global minimum at

$$
\zeta_{0}=\frac{\tau}{k_{2}^{(h)}-k_{1}^{(h)}} \log \left[\left(\frac{\delta_{h}}{\Gamma_{h}}\right)^{\left(k_{2}^{(h)}-k_{1}^{(h)}\right) / k_{1}^{(h)}} \frac{h_{m} k_{2}^{(h)}}{\Gamma_{h} k_{1}^{(h)}}\right]>0 .
$$

Therefore, we get a lower bound of $H(x, t)$ after some calculations, that is,

$$
H(x, t) \geq \min \left\{h_{m}\left(\frac{\delta_{h}}{\Gamma_{h}}\right)^{k_{2}^{(h)} / k_{1}^{(h)}}, \delta_{h} \frac{k_{1}^{(h)}}{k_{2}^{(h)}}\right\} .
$$

Having obtained a lower bound, the rest of the proof of Theorem 1.2 is exactly the same as that of Theorem 1.1.

\subsection{Proof of Proposition 1.3}

To verify Proposition 1.3, we follow the proof of Lemma 3.9 in [8] where the ODE case was treated. We use the assumption $H^{q}>\rho_{a} A^{p-1} / \mu_{a}$ and (1.9) to show that $\tau H_{t} \leq D \boldsymbol{\Lambda}_{h} H-H\left(k_{1}^{(h)}-c H^{\alpha}\right)$ for some positive constants $c$ and $\alpha$. Since $H \rightarrow 0$ as $t \rightarrow+\infty$, for any $\delta_{0}>0$ there is a $T>0$ such that $0<c H^{\alpha} \leq \delta_{0}$ for $t \geq T$, so that $\tau H_{t} \leq D \boldsymbol{\Lambda}_{h} H-\left(k_{1}^{(h)}-\delta_{0}\right) H$. Thus, we obtain the decay estimate: $0<H(x, t) \leq C e^{-\left(k_{1}^{(h)}-\delta_{0}\right) t / \tau}$ for $t>T$. From this and the assumption of the proposition, we have $A(x, t) \leq$ const. $H^{q /(p-1)} \leq$ const. $e^{-\left(k_{1}^{(a)}-\delta_{0}\right) q t /[\tau(p-1)]}$. On the other hand, Lemma 2.1 yields a lower bound: $A(x, t) \geq$ const. $e^{-k_{2}^{(a)} t}$. Comparing these bounds, we conclude that $-k_{2}^{(a)}+$ $q\left(k_{1}^{(h)}-\delta_{0}\right) /[\tau(p-1)] \leq 0$. Since $\delta_{0}$ is arbitrary, we obtain the assertion.

\section{Almost decoupled patterns}

\subsection{Existence}

In this section we prove Theorem 1.5. We start with preparing some symbols which are used throughout this section. Letting $\Sigma_{a, \varepsilon}(x)$ and $\Sigma_{h, D}(x)$ 
be solutions of (1.11) and (1.12), we also use the notation of (1.14). Since $\max _{x \in \bar{\Omega}} \sigma_{a}(x)>0$ and $\max _{x \in \bar{\Omega}} \sigma_{h}(x)>0$, it is clear by the maximum principle that

$$
\left\{\begin{array}{l}
\Sigma_{a, \varepsilon}(x)>0 \quad \text { and } \quad\left\|\Sigma_{a, \varepsilon}\right\|_{L^{\infty}} \leq \max _{x \in \bar{\Omega}} \sigma_{a}(x) / k_{1}^{(a)} \\
\Sigma_{h, D}(x) \geq \delta_{h}>0 .
\end{array}\right.
$$

Here $\delta_{h}$ depends only on $D, \boldsymbol{\Lambda}_{h}, \mu_{h}$ and $\sigma_{h}$.

Fix $\varepsilon>0$ and $D>0$. For simplicity, we put $m_{a}=\max _{x \in \bar{\Omega}} \sigma_{a}(x) / k_{1}^{(a)}$. Let $(A(x), H(x))$ be a stationary solution of (1.1)-(1.3). Putting $A=\Sigma_{a, \varepsilon}+\phi$, $H=\Sigma_{h, D}+\psi$, we seek a solution $(\phi(x), \psi(x))$ of the following equations:

$$
\left\{\begin{array}{l}
\varepsilon^{2} \boldsymbol{\Lambda}_{a} \phi-\mu_{a}(x) \phi+F_{A}^{0} \phi+F_{H}^{0} \psi=-F^{0}-R_{1}(\phi, \psi), \\
D \boldsymbol{\Lambda}_{h} \psi-\mu_{h}(x) \psi+G_{A}^{0} \phi+G_{H}^{0} \psi=-G^{0}-R_{2}(\phi, \psi)
\end{array}\right.
$$

with boundary conditions $\boldsymbol{B}_{a} \phi=\boldsymbol{B}_{h} \psi=0$. Here we have set

$$
\begin{aligned}
& F^{0}=\rho_{a}\left(\sum_{a, \varepsilon}, \Sigma_{h, D}, x\right) \frac{\sum_{a, \varepsilon}^{p}}{\sum_{h, D}^{q}}, \\
& F_{A}^{0}=\frac{\partial \rho_{a}}{\partial A}\left(\sum_{a, \varepsilon}, \Sigma_{h, D}, x\right) \frac{\sum_{a, \varepsilon}^{p}}{\sum_{h, D}^{q}}+\rho_{a}\left(\Sigma_{a, \varepsilon}, \Sigma_{h, D}, x\right) p \frac{\sum_{a, \varepsilon}^{p-1}}{\sum_{h, D}^{q}}, \\
& F_{H}^{0}=\frac{\partial \rho_{a}}{\partial H}\left(\sum_{a, \varepsilon}, \Sigma_{h, D}, x\right) \frac{\sum_{a, \varepsilon}^{p}}{\sum_{h, D}^{q}}-\rho_{a}\left(\Sigma_{a, \varepsilon}, \Sigma_{h, D}, x\right) q \frac{\sum_{a, \varepsilon}^{p}}{\sum_{h, D}^{q+1}}
\end{aligned}
$$

and $G^{0}, G_{A}^{0}$ and $G_{H}^{0}$ are defined by the same formulas as above but $\rho_{a}, p$ and $q$ are replaced by $\rho_{h}, r$ and $s$, respectively. Moreover, $R_{1}(\phi, \psi)$ and $R_{2}(\phi, \psi)$ are defined by

$$
\begin{aligned}
& R_{1}(\phi, \psi)=\rho_{a}\left(\Sigma_{a, \varepsilon}+\phi, \Sigma_{h, D}+\psi, x\right) \frac{\left(\Sigma_{a, \varepsilon}+\phi\right)^{p}}{\left(\Sigma_{h, D}+\psi\right)^{q}}-\left(F^{0}+F_{A}^{0} \phi+F_{H}^{0} \psi\right), \\
& R_{2}(\phi, \psi)=\rho_{h}\left(\Sigma_{a, \varepsilon}+\phi, \Sigma_{h, D}+\psi, x\right) \frac{\left(\Sigma_{a, \varepsilon}+\phi\right)^{r}}{\left(\Sigma_{h, D}+\psi\right)^{s}}-\left(G^{0}+G_{A}^{0} \phi+G_{H}^{0} \psi\right) .
\end{aligned}
$$

Define operators $\mathscr{L}_{\varepsilon}$ and $\mathscr{L}_{D}$ by

$$
\mathscr{L}_{\varepsilon}=L_{\varepsilon}+F_{A}^{0} \quad \text { and } \quad \mathscr{L}_{D}=L_{D}+G_{H}^{0},
$$

where $L_{\varepsilon}=\varepsilon^{2} \boldsymbol{\Lambda}_{a}-\mu_{a}(x)$ and $L_{D}=D \boldsymbol{\Lambda}_{h}-\mu_{h}(x)$. Since $L_{\varepsilon}$ and $L_{D}$ are invertible, we can prove by the maximum principle that

$$
\left\|L_{\varepsilon}^{-1}\right\|_{\mathcal{B}\left(C^{0}(\bar{\Omega})\right)} \leq 1 / k_{1}^{(a)}, \quad\left\|L_{D}^{-1}\right\|_{\mathcal{B}\left(C^{0}(\bar{\Omega})\right)} \leq 1 / k_{1}^{(h)} .
$$

Using these estimates, we will show the following lemma. 
Lemma 3.1. The operators $\mathscr{L}_{\varepsilon}$ and $\mathscr{L}_{D}$ are invertible if $m_{a}$ is sufficiently small. Moreover, we have

$$
\left\|\mathscr{L}_{\varepsilon}^{-1}\right\|_{\mathcal{B}\left(C^{0}(\bar{\Omega})\right)} \leq 2 / k_{1}^{(a)}
$$

if $m_{a}<1$ and $m_{a}^{p-1}<\delta_{h}^{q} k_{1}^{(a)} /\left(2 p C_{a}\right)$. On the other hand,

$$
\left\|\mathscr{L}_{D}^{-1}\right\|_{\mathcal{B}\left(C^{0}(\bar{\Omega})\right)} \leq 2 / k_{1}^{(h)}
$$

provided $m_{a}^{r}<\delta_{h}^{s+1} k_{1}^{(h)} /\left(2 C_{h}\left(\delta_{h}+s\right)\right)$.

Proof. We verify the invertibility of $\mathscr{L}_{\varepsilon}$ and the estimate (3.9). As to $\mathscr{L}_{D}$, the same argument leads to (3.10).

Since we have

$$
\mathscr{L}_{\varepsilon} \phi=L_{\varepsilon}\left(\phi+L_{\varepsilon}^{-1}\left[F_{A}^{0} \phi\right]\right)
$$

for $\phi \in C^{2}(\Omega) \cap C^{1}(\bar{\Omega}), \quad \mathscr{L}_{\varepsilon}$ is invertible if $\left\|L_{\varepsilon}^{-1}\left[F_{A}^{0} \cdot\right]\right\|_{\mathcal{B}\left(C^{0}(\bar{\Omega})\right)}<1$. We obtain from (1.5), (3.1), (3.4) and (3.8) that

$$
\left\|L_{\varepsilon}^{-1}\left[F_{A}^{0} \phi\right]\right\|_{L^{\infty}} \leq \frac{C_{a} m_{a}^{p-1}}{k_{1}^{(a)} \delta_{h}^{q}}\left(m_{a}+p\right)\|\phi\|_{L^{\infty}} .
$$

Letting $m_{a}<1$, we see that $m_{a}<p$ because of $p>1$. Therefore, we have

$$
\left\|L_{\varepsilon}^{-1}\left[F_{A}^{0} \phi\right]\right\|_{L^{\infty}} \leq 2 p \frac{C_{a} m_{a}^{p-1}}{k_{1}^{(a)} \delta_{h}^{q}}\|\phi\|_{L^{\infty}},
$$

which implies that the assertion holds if $m_{a}^{p-1}<\delta_{h}^{q} k_{1}^{(a)} /\left(2 p C_{a}\right)$.

In the following, we assume that $m_{a}$ is sufficiently small so that both (3.9) and (3.10) hold. We introduce complete metric spaces $X$ and $Y$ for $m_{a}$ sufficiently small:

$$
X=\left\{\phi \in C^{0}(\bar{\Omega}) \mid\|\phi\|_{X} \leq \kappa\right\}, \quad Y=\left\{\psi \in C^{0}(\bar{\Omega}) \mid\|\psi\|_{Y} \leq \kappa\right\},
$$

where $\|\phi\|_{X}=\|\phi\|_{L^{\infty}} / m_{a}^{p}$ and $\|\psi\|_{Y}=\|\psi\|_{L^{\infty}} / m_{a}^{r}$. Moreover, $\kappa$ is a positive constant to be fixed later, and $m_{a}$ is taken so small that $\kappa m_{a}^{r}<\delta_{h} / 3$. Hence, if $\psi \in Y$, then

$$
\Sigma_{h, D}(x)+\psi(x)>\frac{2 \delta_{h}}{3} \quad \text { for all } x \in \bar{\Omega}
$$

Defining a mapping $\mathcal{F}(\phi, \psi)=\left(\mathcal{F}_{1}(\phi, \psi), \mathcal{F}_{2}(\phi, \psi)\right)$ from $\quad X \times Y$ into $C^{0}(\bar{\Omega}) \times C^{0}(\bar{\Omega})$ by

$$
\begin{aligned}
& \mathcal{F}_{1}(\phi, \psi)=\mathscr{L}_{\varepsilon}^{-1}\left[-F^{0}-R_{1}(\phi, \psi)-F_{H}^{0} \psi\right], \\
& \mathcal{F}_{2}(\phi, \psi)=\mathscr{L}_{D}^{-1}\left[-G^{0}-R_{2}(\phi, \psi)-G_{A}^{0} \phi\right],
\end{aligned}
$$


we see that if $\left(\phi_{*}, \psi_{*}\right)$ is a fixed point of $\mathcal{F}(\phi, \psi)$, then it is a solution of (3.2). Indeed, since $\mathscr{L}_{\varepsilon}^{-1}$ maps $L^{P}(\Omega)$ into $W_{N}^{2, P}(\Omega)=\left\{\phi \in W^{2, P}(\Omega)\left|\boldsymbol{B}_{a} \phi\right|_{\partial \Omega}=0\right\}$ for any $1<P<\infty$, we see that $\phi_{*} \in C^{1+\alpha}(\bar{\Omega})$ by the Sobolev embedding theorem by taking $P>N$, where $0<\alpha<1$. Similarly, $\psi_{*} \in C^{1+\alpha}(\bar{\Omega})$. Hence the functions $-F^{0}-R_{1}(\phi, \psi)-F_{H}^{0} \psi$ and $-G^{0}-R_{2}(\phi, \psi)-G_{A}^{0} \phi$ are both Hölder continuous on $\bar{\Omega}$. Therefore, the standard elliptic regularity theory implies that $\left(\phi_{*}, \psi_{*}\right)$ is a classical solution of (3.2).

We have the following estimates:

Lemma 3.2. Assume $r \geq 1$. Let $\phi, \hat{\phi} \in X$ and $\psi, \hat{\psi} \in Y$. There is a positive constant $C(\kappa, \rho)$ depending on $\kappa, C_{a}$ and $C_{h}$ besides $(p, q, r, s)$ such that

$$
\begin{aligned}
& \left\|R_{1}(\phi, \psi)\right\|_{L^{\infty}} \leq C(\kappa, \rho) C\left(\delta_{h}\right) c\left(m_{a}\right) m_{a}^{p}, \\
& \left\|R_{2}(\phi, \psi)\right\|_{L^{\infty}} \leq C(\kappa, \rho) C\left(\delta_{h}\right) c\left(m_{a}\right) m_{a}^{r}, \\
& \left\|R_{1}(\phi, \psi)-R_{1}(\hat{\phi}, \hat{\psi})\right\|_{L^{\infty}} \\
& \quad \leq C(\kappa, \rho) C\left(\delta_{h}\right)\left(m_{a}^{p-1}\|\phi-\hat{\phi}\|_{L^{\infty}}+m_{a}^{p}\|\psi-\hat{\psi}\|_{L^{\infty}}\right), \\
& \quad\left\|R_{2}(\phi, \psi)-R_{2}(\hat{\phi}, \hat{\psi})\right\|_{L^{\infty}} \\
& \quad \leq C(\kappa, \rho) C\left(\delta_{h}\right)\left(m_{a}^{r-1}\|\phi-\hat{\phi}\|_{L^{\infty}}+m_{a}^{r}\|\psi-\hat{\psi}\|_{L^{\infty}}\right) .
\end{aligned}
$$

Here, $C\left(\delta_{h}\right)$ and $c\left(m_{a}\right)$ are positive constants depending only on $\delta_{h}$ and on $m_{a}$, respectively, in addition to $(p, q, r, s)$. Moreover, $c\left(m_{a}\right)$ tends to 0 as $m_{a} \downarrow 0$.

Lemma 3.2 can be proved by using (3.1), (3.6), (3.7), (3.11) and the following lemma.

Lemma 3.3. Let $\alpha>0, \beta>0, \phi \in X$ and $\psi \in Y$. Then we have

$$
\left\|\frac{\left(\sum_{a, \varepsilon}+\phi\right)^{\alpha}}{\left(\sum_{h, D}+\psi\right)^{\beta}}-\frac{\sum_{a, \varepsilon}^{\alpha}}{\sum_{h, D}^{\beta}}\right\|_{L^{\infty}} \leq C\left(\delta_{h}\right) C(\kappa)\left(m_{a}^{(p-1) \alpha}+m_{a}^{\beta_{*} r}\right) m_{a}^{\alpha},
$$

where $\beta_{*}=\min \{\beta, 1\}$, and $C(\kappa)$ is a positive constant depending only on $\alpha, \beta$ and $\kappa$.

This lemma follows easily from the following elementary estimates:

$$
\left|a_{+}^{\lambda}-b_{+}^{\lambda}\right| \leq\left\{\begin{array}{l}
\lambda \max \left\{a_{+}^{\lambda-1}, b_{+}^{\lambda-1}\right\}\left|a_{+}-b_{+}\right| \quad \text { if } \lambda \geq 1, \\
\left|a_{+}-b_{+}\right|^{\lambda} \quad \text { if } 0<\lambda<1
\end{array}\right.
$$

in which $a_{+}=\max \{0, a\}$.

Now we start to verify that $\mathcal{F}$ is a contraction mapping. For $\phi, \hat{\phi} \in X$ and $\psi, \hat{\psi} \in Y$, we define $\|(\phi, \psi)-(\hat{\phi}, \hat{\psi})\|_{X \times Y}=\|\phi-\hat{\phi}\|_{X}+\|\psi-\hat{\psi}\|_{Y}$. 
By (3.13), we see that

$$
\begin{aligned}
\left\|\mathcal{F}_{1}(\phi, \psi)\right\|_{L^{\infty}} & \leq \frac{2}{k_{1}^{(a)}}\left(\left\|F_{H}^{0}\right\|_{L^{\infty}}\|\psi\|_{L^{\infty}}+\left\|F^{0}\right\|_{L^{\infty}}+\left\|R_{1}(\phi, \psi)\right\|_{L^{\infty}}\right) \\
& \leq \frac{2}{k_{1}^{(a)}}\left[\frac{C_{a}}{\delta_{h}^{q}}\left\{\left(1+\frac{q}{\delta_{h}}\right) \kappa m_{a}^{r}+1\right\} m_{a}^{p}+C(\kappa, \rho) C\left(\delta_{h}\right) c\left(m_{a}\right) m_{a}^{p}\right] .
\end{aligned}
$$

Hence

$$
\frac{\left\|\mathcal{F}_{1}(\phi, \psi)\right\|_{L^{\infty}}}{m_{a}^{p}} \leq \frac{\kappa}{2}\left[\left(1+\frac{q}{\delta_{h}}\right) \kappa m_{a}^{r}+1\right]+\frac{2}{k_{1}^{(a)}} C(\kappa, \rho) C\left(\delta_{h}\right) c\left(m_{a}\right),
$$

where $\kappa=4 C_{a} /\left(k_{1}^{(a)} \delta_{h}^{q}\right)$. Therefore, if we choose $m_{a}$ so small that

$$
\left(1+\frac{q}{\delta_{h}}\right) \kappa m_{a}^{r} \leq \frac{1}{2} \quad \text { and } \quad \frac{2}{k_{1}^{(a)}} C(\kappa, \rho) C\left(\delta_{h}\right) c\left(m_{a}\right) \leq \frac{\kappa}{4},
$$

then $F_{1}(\phi, \psi) \in X$. Similarly, from (3.14), we have

$$
\left\|\mathcal{F}_{2}(\phi, \psi)\right\|_{L^{\infty}} \leq \frac{2}{k_{1}^{(h)}}\left[\frac{C_{h}}{\delta_{h}^{s}}\left\{\left(m_{a}^{p}+r m_{a}^{p-1}\right) \kappa+1\right\} m_{a}^{r}+C(\kappa, \rho) C\left(\delta_{h}\right) c\left(m_{a}\right) m_{a}^{r}\right] .
$$

We choose $\kappa=\max \left\{4 C_{a} /\left(k_{1}^{(a)} \delta_{h}^{q}\right), 4 C_{h} /\left(k_{1}^{(h)} \delta_{h}^{s}\right)\right\}$ to obtain

$$
\frac{\left\|\mathcal{F}_{2}(\phi, \psi)\right\|_{L^{\infty}}}{m_{a}^{r}} \leq \frac{\kappa}{2}\left[\left(m_{a}^{p}+r m_{a}^{p-1}\right) \kappa+1\right]+\frac{2}{k_{1}^{(h)}} C(\kappa, \rho) C\left(\delta_{h}\right) c\left(m_{a}\right) .
$$

Hence, $\mathcal{F}_{2}(\phi, \psi) \in Y$ if $m_{a}$ satisfies

$$
\left(m_{a}^{p}+r m_{a}^{p-1}\right) \kappa \leq \frac{1}{2} \quad \text { and } \quad \frac{2}{k_{1}^{(h)}} C(\kappa, \rho) C\left(\delta_{h}\right) c\left(m_{a}\right) \leq \frac{\kappa}{4} .
$$

We thus have verified that $\mathcal{F}$ maps $X \times Y$ into itself.

Next, it follows from (3.15) that

$$
\begin{aligned}
\left\|\mathcal{F}_{1}(\phi, \psi)-\mathcal{F}_{1}(\hat{\phi}, \hat{\psi})\right\|_{L^{\infty}} \leq & \left\|\mathscr{L}_{\varepsilon}^{-1}\left[F_{H}^{0}(\psi-\hat{\psi})\right]\right\|_{L^{\infty}}+\left\|\mathscr{L}_{\varepsilon}^{-1}\left[R_{1}(\phi, \psi)-R_{1}(\hat{\phi}, \hat{\psi})\right]\right\|_{L^{\infty}} \\
\leq & \frac{2}{k_{1}^{(a)}}\left(C_{a} \frac{m_{a}^{p}}{\delta_{h}^{q}}+C_{a} q \frac{m_{a}^{p}}{\delta_{h}^{q+1}}\right)\|\psi-\hat{\psi}\|_{L^{\infty}} \\
& +\frac{2}{k_{1}^{(a)}} C(\kappa, \rho) C\left(\delta_{h}\right)\left(m_{a}^{p-1}\|\phi-\hat{\phi}\|_{L^{\infty}}+m_{a}^{p}\|\psi-\hat{\psi}\|_{L^{\infty}}\right) .
\end{aligned}
$$


Therefore, we have

$$
\begin{aligned}
& \frac{\left\|\mathcal{F}_{1}(\phi, \psi)-\mathcal{F}_{1}(\hat{\phi}, \hat{\psi})\right\|_{L^{\infty}}}{m_{a}^{p}} \\
& \leq \frac{2}{k_{1}^{(a)}}\left(C_{a} \frac{1}{\delta_{h}^{q}}+C_{a} q \frac{1}{\delta_{h}^{q+1}}\right) \frac{\|\psi-\hat{\psi}\|_{L^{\infty}}}{m_{a}^{r}} m_{a}^{r} \\
& \quad+\frac{2}{k_{1}^{(a)}} C(\kappa, \rho) C\left(\delta_{h}\right)\left(m_{a}^{p-1} \frac{\|\phi-\hat{\phi}\|_{L^{\infty}}}{m_{a}^{p}}+m_{a}^{r} \frac{\|\psi-\hat{\psi}\|_{L^{\infty}}}{m_{a}^{r}}\right),
\end{aligned}
$$

which implies

$$
\left\|\mathcal{F}_{1}(\phi, \psi)-\mathcal{F}_{1}(\hat{\phi}, \hat{\psi})\right\|_{X} \leq \frac{1}{2}\left(\|\phi-\hat{\phi}\|_{X}+\|\psi-\hat{\psi}\|_{Y}\right)
$$

provided $m_{a}$ is sufficiently small. Similarly, we obtain, by (3.16), that

$$
\begin{aligned}
& \left\|\mathcal{F}_{2}(\phi, \psi)-\mathcal{F}_{2}(\hat{\phi}, \hat{\psi})\right\|_{Y} \\
& \quad \leq\left(\left\|\mathscr{L}_{D}^{-1}\left[G_{A}^{0}(\phi-\hat{\phi})\right]\right\|_{L^{\infty}}+\left\|\mathscr{L}_{D}^{-1}\left[R_{2}(\phi, \psi)-R_{2}(\hat{\phi}, \hat{\psi})\right]\right\|_{L^{\infty}}\right) / m_{a}^{r} \\
& \quad \leq C\left(m_{a}^{p-1}\|\phi-\hat{\phi}\|_{X}+m_{a}^{r}\|\psi-\hat{\psi}\|_{Y}\right) \\
& \quad \leq \frac{1}{2}\left(\|\phi-\hat{\phi}\|_{X}+\|\psi-\hat{\psi}\|_{Y}\right)
\end{aligned}
$$

for $m_{a}$ sufficiently small, where $C$ is a positive constant independent of $m_{a}$. Therefore, we see that $\mathcal{F}$ is a contraction mapping in $X \times Y$. Consequently, there exists a unique fixed point $\left(\phi_{*}, \psi_{*}\right)$ of $\mathcal{F}$ and $\left(A_{*}, H_{*}\right)=$ $\left(\Sigma_{a, \varepsilon}+\phi_{*}, \Sigma_{h, D}+\psi_{*}\right)$ gives a stationary solution of (1.1)-(1.3) with $\left\|\phi_{*}\right\|_{L^{\infty}} \leq$ $\kappa m_{a}^{p}$ and $\left\|\psi_{*}\right\|_{L^{\infty}} \leq \kappa m_{a}^{r}$.

Next we turn to the case $0<r<1$. We assume that $\min _{x \in \bar{\Omega}} \sigma_{a}(x)>$ $3 \kappa k_{2}^{(a)} m_{a}^{p}$. By the maximum principle, $\Sigma_{a, \varepsilon}(x)>3 \kappa m_{a}^{p}$ for all $x \in \bar{\Omega}$. Note that $m_{a}>m_{a}^{p}$ since $m_{a}<1$ and $p>1$. Therefore, for $\phi$ and $\psi$ which satisfy $\|\phi\|_{L^{\infty}} \leq \kappa m_{a}^{p}$ and $\|\psi\|_{L^{\infty}} \leq \kappa m_{a}^{r}$, we see that

$$
\begin{aligned}
& \Sigma_{a, \varepsilon}(x)+t \phi(x) \geq 2 \kappa m_{a}^{p}, \\
& \Sigma_{h, D}(x)+t \psi(x) \geq \delta_{h}-\kappa m_{a}^{r} \geq \frac{2 \delta_{h}}{3} \quad(x \in \bar{\Omega}, 0 \leq t \leq 1) .
\end{aligned}
$$

Under these assumptions, the same line of arguments as in the case $r \geq 1$ can be applied. Therefore, we see that $\mathcal{F}$ becomes a contraction mapping in $X \times Y$ by using (3.20) and the following lemma: 
Lemma 3.4. Let $0<\alpha<1, \beta>0, \phi \in X$ and $\psi \in Y$. Then we have

$$
\left\|\frac{1}{\left(\sum_{a, \varepsilon}+\phi\right)^{\alpha}\left(\Sigma_{h, D}+\psi\right)^{\beta}}-\frac{1}{\sum_{a, \varepsilon}^{\alpha} \sum_{h, D}^{\beta}}\right\|_{L^{\infty}} \leq C\left(\delta_{h}\right) C(\kappa) \frac{1+m_{a}^{\beta_{*} r}}{m_{a}^{\alpha p}},
$$

where $\beta_{*}=\min \{\beta, 1\}, C(\kappa)$ is a positive constant depending on $\kappa$.

The estimate of $R_{2}(\phi, \psi)-R_{2}(\hat{\phi}, \hat{\psi})$ is delicate because this contains the factor $\left(A_{*}+\phi\right)^{r-1}$. To avoid this difficulty we use the weighted norm $\|\cdot\|_{Y}$ instead of the $L^{\infty}$-norm in (3.19), which we handle as follows:

$$
\begin{aligned}
\mid R_{2}(\phi, \psi) & -R_{2}(\hat{\phi}, \hat{\psi}) \mid \\
\leq \| & \left(\rho_{h}\left(\Sigma_{a, \varepsilon}+\phi, \Sigma_{h, D}+\psi, x\right)\right. \\
& \left.-\rho_{h}\left(\Sigma_{a, \varepsilon}+\hat{\phi}, \Sigma_{h, D}+\hat{\psi}, x\right)\right) \frac{\left(\Sigma_{a, \varepsilon}+\phi\right)^{r}}{\left(\Sigma_{h, D}+\psi\right)^{s}} \\
& +\rho_{h}\left(\sum_{a, \varepsilon}+\hat{\phi}, \Sigma_{h, D}+\hat{\psi}, x\right)\left(\frac{\left(\Sigma_{a, \varepsilon}+\phi\right)^{r}}{\left(\Sigma_{h, D}+\psi\right)^{s}}-\frac{\left(\Sigma_{a, \varepsilon}+\hat{\phi}\right)^{r}}{\left(\Sigma_{h, D}+\hat{\psi}\right)^{s}}\right) \\
& -\frac{\partial \rho_{h}}{\partial A}\left(\Sigma_{a, \varepsilon}, \Sigma_{h, D}, x\right) \frac{\Sigma_{a, \varepsilon}^{r}}{\sum_{h, D}^{s}}(\phi-\hat{\phi}) \\
& -r \rho_{h}\left(\sum_{a, \varepsilon}, \Sigma_{h, D}, x\right) \frac{\sum_{a, \varepsilon}^{r-1}}{\sum_{h, D}^{s}}(\phi-\hat{\phi})-G_{H}^{0}(\psi-\hat{\psi}) \|_{L^{\infty}} .
\end{aligned}
$$

Since our problem is to estimate the terms with the exponent $r-1$, we consider the second and fourth terms, $I_{2}$ and $I_{4}$, of the right hand side of (3.21). By the mean value theorem we obtain

$$
\begin{aligned}
\left\|I_{2}+I_{4}\right\|_{L^{\infty}} \leq & r C_{h} \int_{0}^{1}\left\|\frac{\left(\sum_{a, \varepsilon}+\hat{\phi}+t(\phi-\hat{\phi})\right)^{r-1}}{\left(\sum_{h, D}+\hat{\psi}+t(\psi-\hat{\psi})\right)^{s}}-\frac{\sum_{a, \varepsilon}^{r-1}}{\sum_{h, D}^{s}}\right\|_{L^{\infty}} d t\|\phi-\hat{\phi}\|_{L^{\infty}} \\
& +r \int_{0}^{1}\left\|\frac{\partial \rho_{h}}{\partial A}\left(\sum_{a, \varepsilon}+t \hat{\phi}, \Sigma_{h, D}+t \hat{\psi}, x\right)\right\|_{L^{\infty}} d t\|\hat{\phi}\|_{L^{\infty}} \frac{\sum_{a, \varepsilon}^{r-1}}{\sum_{h, D}^{s}}\|\phi-\hat{\phi}\|_{L^{\infty}} \\
& +r \int_{0}^{1}\left\|\frac{\partial \rho_{h}}{\partial H}\left(\sum_{a, \varepsilon}+t \hat{\phi}, \Sigma_{h, D}+t \hat{\psi}, x\right)\right\|_{L^{\infty}} d t\|\hat{\psi}\|_{L^{\infty}} \frac{\sum_{a, \varepsilon}^{r-1}}{\sum_{h, D}^{s}}\|\phi-\hat{\phi}\|_{L^{\infty}} .
\end{aligned}
$$

Noting that $r-1<0$, we see from Lemma 3.4 that 


$$
\begin{aligned}
\left\|I_{2}+I_{4}\right\|_{L^{\infty}} \leq & C\left(\delta_{h}\right) C(\kappa, \rho) \frac{1+m_{a}^{s_{*} r}}{m_{a}^{p(1-r)}}\|\phi-\hat{\phi}\|_{L^{\infty}}+C\left(\delta_{h}\right) C(\kappa, \rho) \frac{m_{a}^{p}}{m_{a}^{p(1-r)}}\|\phi-\hat{\phi}\|_{L^{\infty}} \\
& +C\left(\delta_{h}\right) C(\kappa, \rho) \frac{m_{a}^{r}}{m_{a}^{p(1-r)}}\|\phi-\hat{\phi}\|_{L^{\infty}} \\
\leq & C\left(\delta_{h}\right) C(\kappa, \rho) m_{a}^{r} m_{a}^{r(p-1)}\left[\left(1+m_{a}^{s_{*} r}\right)+m_{a}^{p}+m_{a}^{r}\right]\|\phi-\hat{\phi}\|_{X} .
\end{aligned}
$$

This implies $\left\|I_{2}+I_{4}\right\|_{Y} \leq C\left(\delta_{h}\right) C(\kappa, \rho) m_{a}^{r(p-1)}\|\phi-\hat{\phi}\|_{X}$. Therefore, combining other estimates, we obtain

$$
\left\|R_{2}(\phi, \psi)-R_{2}(\hat{\phi}, \hat{\psi})\right\|_{Y} \leq C\left(\delta_{h}\right) C(\kappa, \rho) c\left(m_{a}\right)\left(\|\phi-\hat{\phi}\|_{X}+\|\psi-\hat{\psi}\|_{Y}\right) .
$$

\subsection{Stability}

We here recall that $A_{*}(x)=\Sigma_{a, \varepsilon}(x)+\phi_{*}(x)$ and $H_{*}(x)=\Sigma_{h, D}(x)+\psi_{*}(x)$; and that $\max _{x \in \bar{\Omega}} \Sigma_{a, \varepsilon}(x) \leq m_{a}, \quad \min _{x \in \bar{\Omega}} \Sigma_{h, D}(x)=\delta_{h},\left\|\phi_{*}\right\|_{L^{\infty}(\Omega)} \leq \kappa m_{a}^{p}$ and $\left\|\psi_{*}\right\|_{L^{\infty}(\Omega)} \leq \kappa m_{a}^{r}$. Note also that $\min _{x \in \bar{\Omega}} \Sigma_{a, \varepsilon}(x) \geq \min _{x \in \bar{\Omega}} \sigma_{a}(x) / k_{2}^{(a)}$ and $\min _{x \in \bar{\Omega}} \psi_{*}(x) \geq 0$. Therefore, we may assume that (i) $\max _{x \in \bar{\Omega}} A_{*}(x) \leq 2 m_{a}$, (ii) $\min _{x \in \bar{\Omega}} H_{*}(x) \geq \delta_{h}$, and (iii) in the case $0<r<1$, there exists a positive constant $c_{A_{*}}$ such that $\min _{x \in \bar{\Omega}} A_{*}(x) \geq c_{A_{*}} m_{a}^{p}$, whenever $\max _{x \in \bar{\Omega}} \sigma_{a}(x) \leq m_{0}$.

The goal in this subsection is to prove the following proposition.

Proposition 3.5. There exists positive constants $m_{l}$ and $K$ such that the following statements hold for $0<m_{a} \leq m_{l}$. Let $C_{\phi}$ and $C_{\psi}$ be positive constants satisfying

$$
3 K\left(C_{\phi}+C_{\psi}\right) \leq\left\{\begin{array}{l}
\min \left\{2, \delta_{h}\right\} \quad \text { if } r \geq 1 \\
\min \left\{c_{A_{*}}, \delta_{h}\right\} \quad \text { if } 0<r<1
\end{array}\right.
$$

If the solution $(A(x, t), H(x, t))$ of the initial-boundary value problem (1.1)-(1.3) satisfies

$$
\begin{aligned}
& \left\|A(\cdot, 0)-A_{*}\right\|_{L^{\infty}} \leq\left\{\begin{array}{ll}
C_{\phi} m_{a}^{p} & \text { if } 0<r<1 \\
C_{\phi} m_{a} & \text { if } r \geq 1
\end{array}\right. \text { and } \\
& \left\|H(\cdot, 0)-H_{*}\right\|_{L^{\infty}} \leq C_{\psi},
\end{aligned}
$$

then

$$
\begin{aligned}
& \left\|A(\cdot, t)-A_{*}\right\|_{L^{\infty}} \leq \begin{cases}2 K\left(C_{\phi}+C_{\psi}\right) m_{a}^{p} e^{-\lambda t} & \text { if } 0<r<1 \\
2 K\left(C_{\phi}+C_{\psi}\right) m_{a} e^{-\lambda t} & \text { if } r \geq 1,\end{cases} \\
& \left\|H(\cdot, t)-H_{*}\right\|_{L^{\infty}} \leq 2 K\left(C_{\phi}+C_{\psi}\right) e^{-\lambda t}
\end{aligned}
$$


for all $t>0$. Here, $\lambda$ is any number satisfying

$$
0<8 \lambda<\min \left\{k_{1}^{(a)}, k_{1}^{(h)} / \tau\right\}
$$

To begin with, we put

$$
A(x, t)=A_{*}(x)+\phi(x, t) \quad \text { and } \quad H(x, t)=H_{*}(x)+\psi(x, t)
$$

and write down the equations satisfied by $(\phi, \psi)$ :

$$
\begin{gathered}
\frac{\partial \phi}{\partial t}=\varepsilon^{2} \boldsymbol{\Lambda}_{a} \phi-\mu_{a} \phi+\rho_{a}\left(A_{*}+\phi, H_{*}+\psi, x\right) \frac{\left(A_{*}+\phi\right)^{p}}{\left(H_{*}+\psi\right)^{q}}-\rho_{a}(*) \frac{A_{*}^{p}}{H_{*}^{q}}, \\
\tau \frac{\partial \psi}{\partial t}=D \boldsymbol{\Lambda}_{h} \psi-\mu_{h} \psi+\rho_{h}\left(A_{*}+\phi, H_{*}+\psi, x\right) \frac{\left(A_{*}+\psi\right)^{r}}{\left(H_{*}+\psi\right)^{s}}-\rho_{h}(*) \frac{A_{*}^{r}}{H_{*}^{s}},
\end{gathered}
$$

where we have defined

$$
\rho_{a}(*)=\rho_{a}\left(A_{*}(x), H_{*}(x), x\right) \quad \text { and } \quad \rho_{h}(*)=\rho_{h}\left(A_{*}(x) H_{*}(x), x\right) .
$$

By the mean value theorem we have

$$
\begin{aligned}
\rho_{a}\left(A_{*}+\phi, H_{*}+\psi, x\right) \frac{\left(A_{*}+\phi\right)^{p}}{\left(H_{*}+\psi\right)^{q}}-\rho_{a}(*) \frac{A_{*}^{p}}{H_{*}^{q}} \\
=\left[\rho_{a}\left(A_{*}+\phi, H_{*}+\psi, x\right)-\rho_{a}\left(A_{*}, H_{*}, x\right)\right] \frac{\left(A_{*}+\phi\right)^{p}}{\left(H_{*}+\psi\right)^{q}}+\rho_{a}(*)\left[\frac{\left(A_{*}+\phi\right)^{p}}{\left(H_{*}+\psi\right)^{q}}-\frac{A_{*}^{p}}{H_{*}^{q}}\right] \\
=\frac{\left(A_{*}+\phi\right)^{p}}{\left(H_{*}+\psi\right)^{q}}\left(\frac{\partial \rho_{a}}{\partial A}\left(A_{*}+\theta_{1} \phi, H_{*}+\theta_{1} \psi, x\right) \phi+\frac{\partial \rho_{h}}{\partial H}\left(A_{*}+\theta_{1} \phi, H_{*}+\theta_{1} \psi, x\right) \psi\right) \\
\quad+\rho_{a}\left(A_{*}+\phi, H_{*}+\psi, x\right)\left[p \frac{\left(A_{*}+\theta_{2} \phi\right)^{p-1}}{\left(H_{*}+\theta_{2} \psi\right)^{q}} \phi-q \frac{\left(A_{*}+\theta_{2} \phi\right)^{p}}{\left(H_{*}+\theta_{2} \psi\right)^{q+1}} \psi\right]
\end{aligned}
$$

with appropriate constants $0<\theta_{1}<1,0<\theta_{2}<1$ and also a similar expression for $\rho_{h}\left(A_{*}+\phi, H_{*}+\psi, x\right)\left(A_{*}+\phi\right)^{r} /\left(H_{*}+\psi\right)^{s}-\rho_{h}(*) A_{*}^{r} / H_{*}^{s}$. Hence, we define

$$
\begin{aligned}
\gamma_{11}(x, t)= & \frac{\left(A_{*}+\phi\right)^{p}}{\left(H_{*}+\psi\right)^{q}} \frac{\partial \rho_{a}}{\partial A}\left(A_{*}+\theta_{1} \phi, H_{*}+\theta_{1} \psi, x\right) \\
& +p \rho_{a}\left(A_{*}+\phi, H_{*}+\psi, x\right) \frac{\left(A_{*}+\theta_{2} \phi\right)^{p-1}}{\left(H_{*}+\theta_{2} \psi\right)^{q}}, \\
\gamma_{12}(x, t)= & \frac{\left(A_{*}+\phi\right)^{p}}{\left(H_{*}+\psi\right)^{q}} \frac{\partial \rho_{a}}{\partial H}\left(A_{*}+\theta_{1} \phi, H_{*}+\theta_{1} \psi, x\right) \\
& -q \rho_{a}\left(A_{*}+\phi, H_{*}+\psi, x\right) \frac{\left(A_{*}+\theta_{2} \phi\right)^{p}}{\left(H_{*}+\theta_{2} \psi\right)^{q+1}},
\end{aligned}
$$




$$
\begin{aligned}
\gamma_{21}(x, t)= & \frac{\left(A_{*}+\phi\right)^{r}}{\left(H_{*}+\psi\right)^{s}} \frac{\partial \rho_{h}}{\partial A}\left(A_{*}+\theta_{3} \phi, H_{*}+\theta_{3} \psi, x\right) \\
& +r \rho_{h}\left(A_{*}+\phi, H_{*}+\psi, x\right) \frac{\left(A_{*}+\theta_{4} \phi\right)^{r-1}}{\left(H_{*}+\theta_{4} \psi\right)^{s}}, \\
\gamma_{22}(x, t)= & \frac{\left(A_{*}+\phi\right)^{r}}{\left(H_{*}+\psi\right)^{q}} \frac{\partial \rho_{h}}{\partial H}\left(A_{*}+\theta_{3} \phi, H_{*}+\theta_{3} \psi, x\right) \\
& -s \rho_{h}\left(A_{*}+\phi, H_{*}+\psi, x\right) \frac{\left(A_{*}+\theta_{4} \phi\right)^{r}}{\left(H_{*}+\theta_{4} \psi\right)^{s+1}}
\end{aligned}
$$

with the understanding that the $\theta_{j}$ 's are constants in the interval $(0,1)$ determined by the mean value theorem.

Then $(\phi, \psi)$ solves the following initial-boundary value problem:

$$
\begin{array}{rlrl}
\frac{\partial \phi}{\partial t} & =\varepsilon^{2} \boldsymbol{\Lambda}_{a} \phi-\left(\mu_{a}-\gamma_{11}\right) \phi+\gamma_{12} \psi & \text { for } x \in \Omega, t>0, \\
\tau \frac{\partial \psi}{\partial t} & =D \boldsymbol{\Lambda}_{h} \psi-\left(\mu_{h}-\gamma_{22}\right) \psi+\gamma_{21} \phi \quad \text { for } x \in \Omega, t>0, \\
\boldsymbol{B}_{a} \phi & =\boldsymbol{B}_{h} \psi=0 \quad \text { for } x \in \partial \Omega, t>0, \\
\phi(x, 0) & =\phi_{0}(x), \quad \psi(x, 0)=\psi_{0}(x) \quad \text { for } x \in \Omega .
\end{array}
$$

First we derive estimates of the coefficients $\gamma_{i j}(i, j=1,2)$.

Lemma 3.6. Let $T$ be an arbitrary positive number, and $\phi(x, t), \psi(x, t)$ be any continuous functions on the closed domain $\bar{\Omega} \times[0, T]$ satisfying

$$
\begin{aligned}
& \|\phi\|_{L^{\infty}(\Omega \times(0, T))} \leq\left\{\begin{array}{l}
2 m_{a} \text { if } r \geq 1 \\
c_{A_{*}} m_{a}^{p} / 2 \quad \text { if } 0<r<1,
\end{array}\right. \text { and } \\
& \|\psi\|_{L^{\infty}(\Omega \times(0, T))} \leq \delta_{h} / 2 .
\end{aligned}
$$

Then there exist positive constants $C_{i j}$, with $i, j=1,2$, independent of $m_{a}$ in the interval $0<m_{a} \leq m_{0}$ such that

(i) $\left|\gamma_{11}(x, t)\right| \leq C_{11} m_{a}^{p-1}$;

(ii) $\left|\gamma_{12}(x, t)\right| \leq C_{12} m_{a}^{p}$;

(iii) $\left|\gamma_{21}(x, t)\right| \leq\left\{\begin{array}{l}C_{21} m_{a}^{r-1} \quad \text { if } r \geq 1 \\ C_{21} m_{a}^{p(r-1)} \quad \text { if } 0<r<1 ;\end{array}\right.$

(iv) $\left|\gamma_{22}(x, t)\right| \leq C_{22} m_{a}^{r}$ for all $x \in \bar{\Omega}$ and $0 \leq t \leq T$. 
Proof. By (3.34), we see that $\left\|A_{*}+\phi\right\|_{L^{\infty}(\Omega)} \leq \max _{x \in \bar{\Omega}} A_{*}(x)+2 m_{a} \leq$ $4 m_{a}$ and $\min _{x \in \bar{\Omega}}\left(H_{*}(x)+\psi(x)\right) \geq \min _{x \in \bar{\Omega}} H_{*}(x)-\delta_{h} / 2 \geq \delta_{h} / 2 . \quad$ In addition, $\min _{x \in \bar{\Omega}}\left(A_{*}(x)+\phi(x)\right) \geq \min _{x \in \bar{\Omega}} A_{*}(x)-c_{A_{*}} m_{a}^{p} / 2 \geq c_{A_{*}} m_{a}^{p} / 2$ if $0<r<1$. Let $0 \leq \vartheta \leq 1$. If $\alpha \geq 0$ and $\beta>0$ then

$$
\frac{\left(A_{*}+\vartheta \phi\right)^{\alpha}}{\left(H_{*}+\vartheta \psi\right)^{\beta}} \leq \frac{\left(4 m_{a}\right)^{\alpha}}{\left(\delta_{h} / 2\right)^{\beta}} \leq 2^{2 \alpha+\beta}\left(\delta_{h}\right)^{-\beta} m_{a}^{\alpha} .
$$

If $\alpha<0$ and $\beta>0$, then

$$
\frac{\left(A_{*}+\vartheta \phi\right)^{\alpha}}{\left(H_{*}+\vartheta \psi\right)^{\beta}} \leq \frac{\left(c_{A_{*}} m_{a}^{p} / 2\right)^{\alpha}}{\left(\delta_{h} / 2\right)^{\beta}}=c_{A_{*}}^{\alpha} \delta_{h}^{-\beta} m_{a}^{\alpha p} .
$$

Combining these observations and assumptions (1.5) and (1.6), we obtain the assertions of the lemma.

First we choose $m_{l}>0$ so small that

$$
C_{11} m_{a}^{p-1} \leq \frac{k_{1}^{(a)}}{2} \quad \text { and } \quad C_{22} m_{a}^{r} \leq \frac{k_{1}^{(h)}}{2}
$$

if $0<m_{a} \leq m_{l}$. Then

$$
\mu_{a}(x)-\gamma_{11}(x, t) \geq \frac{k_{1}^{(a)}}{2} \quad \text { and } \quad \mu_{h}(x)-\gamma_{22}(x, t) \geq \frac{k_{1}^{(h)}}{2}
$$

for all $x \in \bar{\Omega}$ and $0 \leq t \leq T$ as long as $\phi$ and $\psi$ satisfy (3.34).

Lemma 3.7. Assume that $m_{l}$ is chosen so that (3.35) holds for $0<m_{a} \leq m_{l}$. Let $(\phi, \psi)$ be a solution of the initial-boundary value problem (3.30)-(3.33). For $\lambda$ satisfying (3.25) put

$$
U(x, t)=e^{\lambda t} \phi(x, t) \quad \text { and } \quad V(x, t)=e^{\lambda t} \psi(x, t) .
$$

If $(\phi, \psi)$ satisfies (3.34) in the domain $\Omega \times(0, T)$ and $0<m_{a} \leq m_{l}$, then

$$
\begin{aligned}
\|U\|_{T} & \leq\left\|\phi_{0}\right\|_{L^{\infty}(\Omega)}+\frac{4 C_{12} m_{a}^{p}}{k_{1}^{(a)}}\|V\|_{T}, \\
\|V\| \|_{T} & \leq\left\|\psi_{0}\right\|_{L^{\infty}(\Omega)}+\frac{4 C_{21} m_{a}^{p(r-1)}}{k_{1}^{(h)}}\|U\|_{T} \quad \text { if } 0<r<1, \\
\|V\|_{T} & \leq\left\|\psi_{0}\right\|_{L^{\infty}(\Omega)}+\frac{4 C_{21} m_{a}^{r-1}}{k_{1}^{(h)}}\|U\|_{T} \quad \text { if } r \geq 1,
\end{aligned}
$$

where $\||f|\|_{T}=\|f\|_{L^{\infty}(\Omega \times(0, T))}$. 
Proof. Multiply both sides of (3.30) by $|\phi|^{\ell-2} \phi$, with $\ell \geq 2$, and then integrate the resulting equation over $\Omega$. After integration by parts one obtains by virtue of the boundary conditions (3.32) that

$$
\begin{aligned}
\frac{1}{\ell} \frac{d}{d t} \int_{\Omega}|\phi|^{\ell} d x= & -\varepsilon^{2}(\ell-1) \int_{\Omega}|\phi|^{\ell-2} \sum_{i, j=1}^{N} d_{i j}^{(a)} \frac{\partial \phi}{\partial x_{i}} \frac{\partial \phi}{\partial x_{j}} d x \\
& -\int_{\Omega}\left(\mu_{a}-\gamma_{11}\right)|\phi|^{\ell} d x+\int_{\Omega} \gamma_{12} \psi|\phi|^{\ell-2} \phi d x \\
\leq & -\varepsilon^{2}(\ell-1) \int_{\Omega}|\phi|^{\ell-2}|\nabla \phi|^{2} d x-\frac{k_{1}^{(a)}}{2} \int_{\Omega}|\phi|^{\ell} d x+\int_{\Omega}\left|\gamma_{12} \psi\right||\phi|^{\ell-1} d x
\end{aligned}
$$

where we have used (3.34) and (3.36). By making use of Hölder's inequality and then Young's inequality, we obtain

$$
\begin{gathered}
\frac{1}{\ell} \frac{d}{d t} \int_{\Omega}|\phi|^{\ell} d x+\varepsilon^{2}(\ell-1) \int_{\Omega}|\phi|^{\ell-2}|\nabla \phi|^{2} d x+\frac{k_{1}^{(a)}}{4} \int_{\Omega}|\phi|^{\ell} d x \\
\leq K_{a}(\ell)^{\ell-1} \int_{\Omega}\left|\gamma_{12} \psi\right|^{\ell} d x, \quad \text { where } K_{a}(\ell)=\frac{4(\ell-1)}{k_{1}^{(a)} \ell} .
\end{gathered}
$$

Therefore,

$$
\begin{aligned}
\int_{\Omega}|\phi(x, t)|^{\ell} d x \leq & \int_{\Omega}\left|\phi_{0}(x)\right|^{\ell} d x e^{k_{1}^{(a)} \ell t / 4} \\
& +\ell K_{a}(\ell)^{\ell-1} \int_{0}^{t} e^{-k_{1}^{(a)} \ell(t-s) / 4} \int_{\Omega}\left|\gamma_{12}(x, t)\right|^{\ell}|\psi(x, t)|^{\ell} d x d t .
\end{aligned}
$$

In exactly the same way, from (3.31) we obtain

$$
\begin{aligned}
& \frac{\tau}{\ell} \frac{d}{d t} \int_{\Omega}|\psi|^{\ell} d x+D(\ell-1) \int_{\Omega}|\psi|^{\ell-2}|\nabla \psi|^{\ell} d x+\frac{k_{1}^{(h)}}{4} \int_{\Omega}|\psi|^{\ell} d x \\
& \quad \leq K_{h}(\ell)^{\ell-1} \int_{\Omega}\left|\gamma_{21} \phi\right|^{\ell} d x, \quad \text { where } K_{h}(\ell)=\frac{4(\ell-1)}{k_{1}^{(h)} \ell}
\end{aligned}
$$

and

$$
\begin{aligned}
\int_{\Omega}|\psi(x, t)|^{\ell} d x \leq & \int_{\Omega}\left|\psi_{0}(x)\right|^{\ell} d x e^{-k_{1}^{(h)} \ell t /(4 \tau)} \\
& +\frac{\ell}{\tau} K_{h}(\ell)^{\ell-1} \int_{0}^{t} e^{-k_{1}^{(a)} \ell(t-s) /(4 \tau)} \int_{\Omega}\left|\gamma_{21}(x, t)\right|^{\ell}|\phi(x, t)|^{\ell} d x d t .
\end{aligned}
$$


Set

$$
U_{\ell}(t)=\int_{\Omega}\left|e^{\lambda t} \phi(x, t)\right|^{\ell} d x \quad \text { and } \quad V_{\ell}(t)=\int_{\Omega}\left|e^{\lambda t} \psi(x, t)\right|^{\ell} d x .
$$

Then, since $-2 \lambda l>-k_{1}^{(a)} \ell / 4$ and $-2 \lambda l>-k_{1}^{(h)} \ell /(4 \tau)$, in view of Lemma 3.6 we have from (3.42) and (3.44) that

$$
\begin{aligned}
& U_{\ell}(t) \leq U_{\ell}(0) e^{-\lambda \ell t}+\ell K_{a}(\ell)^{\ell-1}\left(C_{12} m_{a}^{p}\right)^{\ell} \int_{0}^{t} e^{-\lambda \ell(t-s)} V_{\ell}(s) d s, \\
& V_{\ell}(t) \leq V_{\ell}(0) e^{-\lambda \ell t}+\frac{\ell}{\tau} K_{h}(\ell)^{\ell-1}\left(C_{21} m_{a}^{p(r-1)}\right)^{l} \int_{0}^{t} e^{-\lambda \ell(t-s)} U_{\ell}(s) d s .
\end{aligned}
$$

From (3.45) it follows that

$$
U_{\ell}(t) \leq U_{\ell}(0) e^{-\lambda \ell t}+\lambda^{-1} K_{a}(\ell)^{\ell-1}\left(C_{12} m_{a}^{p}\right)^{\ell} \sup _{0<s<T} V_{\ell}(s)
$$

for all $0 \leq t \leq T$. This implies that

$$
\sup _{0<t<T} U_{\ell}(t)^{1 / \ell} \leq U_{\ell}(0)^{1 / \ell}+\lambda^{-1 / \ell} K_{a}(\ell)^{(\ell-1) / \ell} C_{12} m_{a}^{p} \sup _{0<s<T} V_{\ell}(s)^{1 / \ell} .
$$

Letting $\ell \rightarrow \infty$, we have

$$
\sup _{x \in \Omega, 0<s<T}|U(x, s)| \leq \sup _{x \in \Omega}|U(x, 0)|+\left[4 / k_{1}^{(a)}\right]\left(C_{12} m_{a}^{p}\right) \sup _{x \in \Omega, 0<s<T}|V(x, s)| .
$$

In a similar way we see from (3.44) that

$$
\begin{aligned}
\sup _{x \in \Omega, 0<s<T}|V(x, s)| \leq & \sup _{x \in \Omega}|V(x, 0)| \\
& +\left[4 / k_{1}^{(h)}\right]\left(C_{21} m_{a}^{p(r-1)}\right) \sup _{x \in \Omega, 0<s<T}|U(x, s)| .
\end{aligned}
$$

This completes the proof for the case $0<r<1$. The proof of the case $r \geq 1$ is the same and hence is omitted.

Proof of Proposition 3.5. We treat the case $0<r<1$ first. Choose $m_{l}>0$ so small that

$$
\frac{16 C_{12} C_{21}}{k_{1}^{(a)} k_{1}^{(h)}} m_{a}^{p r} \leq \frac{1}{2} \quad \text { and } \quad \frac{4 C_{21}}{k_{1}^{(a)}} m_{a}^{p r} \leq \frac{1}{2} \quad \text { for } 0<m_{a} \leq m_{l} .
$$

Put

$$
K=\max \left\{\frac{4 C_{12}}{k_{1}^{(h)}}, 2\right\}
$$


and assume that positive constants $C_{\phi}$ and $C_{\psi}$ satisfy

$$
3 K\left(C_{\phi}+C_{\psi}\right) \leq \min \left\{c_{A_{*}}, \delta_{h}\right\}
$$

We claim that if

$$
\left\|\phi_{0}\right\|_{L^{\infty}} \leq C_{\phi} m_{a}^{p} \quad \text { and } \quad\left\|\psi_{0}\right\|_{L^{\infty}} \leq C_{\psi},
$$

then

$$
\begin{aligned}
& \sup _{x \in \Omega, 0<t<+\infty}\left|e^{\lambda t} \phi(x, t)\right| \leq 2 K\left(C_{\phi}+C_{\psi}\right) m_{a}^{p} \quad \text { and } \\
& \sup _{x \in \Omega, 0<t<+\infty}\left|e^{\lambda t} \psi(x, t)\right| \leq 2 K\left(C_{\phi}+C_{\psi}\right) .
\end{aligned}
$$

Since $K \geq 2$, we see that $U(x, t)$ satisfies $\sup _{x \in \Omega}|U(x, t)|<K C_{\phi} m_{a}^{p}$ and $\sup _{x \in \Omega}|V(x, t)|<K C_{\psi}$ for $t>0$ sufficiently small. Hence we assume that there exists a positive number $T$ such that

$$
\begin{aligned}
& \sup _{x \in \Omega}|U(x, t)|<2 K\left(C_{\phi}+C_{\psi}\right) m_{a}^{p}, \\
& \sup _{x \in \Omega}|V(x, t)|<2 K\left(C_{\phi}+C_{\psi}\right) \quad \text { for } 0 \leq t<T
\end{aligned}
$$

and

$$
\sup _{x \in \Omega}|U(x, T)|=2 K\left(C_{\phi}+C_{\psi}\right) m_{a}^{p} \quad \text { or } \quad \sup _{x \in \Omega}|V(x, T)|=2 K\left(C_{\phi}+C_{\psi}\right) .
$$

Bound $\||U|\|_{T}$ on the right-hand side of (3.39) by (3.38) and then use (3.49). We thus obtain

$$
\|V\|_{T} \leq 2 C_{\psi}+C_{\phi}
$$

From (3.56) and (3.38) follows that

$$
\|U\|_{T} \leq\left((1+K) C_{\phi}+2 K C_{\psi}\right) m_{a}^{p} .
$$

Since $K>1$, we see that $\|U\|_{T}<2 K\left(C_{\phi}+C_{\psi}\right) m_{a}^{p}$ and $\|V\| \|_{T}<2 K\left(C_{\phi}+C_{\psi}\right)$. This is a contradiction. Therefore, we conclude that (3.53) holds true.

In the case where $r \geq 1$ we argue along the same line as above. Inequality (3.39) is replaced by (3.40), and by making use of these estimates, we can relax the condition on the smallness of the initial data $\phi_{0}$ considerably. In place of $A_{*}+\phi$ in (3.26)-(3.29) we use $\left(A_{*}+\phi\right)_{+}=\max \left\{A_{*}+\phi, 0\right\}$. Obviously, Lemma 3.6 still holds in the case $r \geq 1$ even after this modification. Let $C_{\phi}, C_{\psi}$ be positive numbers satisfying

$$
3\left(C_{\phi}+C_{\psi}\right) \leq \min \left\{2, \delta_{h}\right\}
$$


We choose $m_{l}$ so small that

$$
\begin{aligned}
& \frac{16 C_{12} C_{21} m_{a}^{p+r-1}}{k_{1}^{(a)} k_{1}^{(h)}} \leq \frac{1}{2}, \quad \frac{4 C_{12} m_{a}^{p-1}}{k_{1}^{(a)}} \leq \frac{1}{2}, \\
& \frac{4 C_{21} m_{a}^{r}}{k_{1}^{(h)}} \leq \frac{1}{2} \quad \text { for } 0<m_{a}<m_{l} .
\end{aligned}
$$

We assume that

$$
\left\|\phi_{0}\right\|_{L^{\infty}(\Omega)} \leq C_{\phi} m_{a} \quad \text { and } \quad\left\|\psi_{0}\right\|_{L^{\infty}(\Omega)} \leq C_{\psi} .
$$

We repeat the argument above with $K=1$ and obtain from (3.40) and (3.38) the estimate

$$
\|V\|_{T} \leq 2 C_{\psi}+C_{\phi}
$$

and then, by making use of (3.58) this time,

$$
\|U \mid\|_{T} \leq\left(\frac{3}{2} C_{\phi}+C_{\psi}\right) m_{a} .
$$

Again we reach a contradiction to (3.55). Thus the proof of the proposition is now complete.

To prove the collapse of patterns in the case where $0<r<1$, one needs to relax the condition on the size of the admissible initial data $\phi_{0}(x)$.

In what follows we assume that $A_{0}(x) \geq \Sigma_{a, \varepsilon}(x)$ and $H_{0}(x) \geq \Sigma_{h, D}(x)$. Then by the maximum principle we see that $A(x, t) \geq \Sigma_{a, \varepsilon}(x)$ and $H(x, t) \geq$ $\Sigma_{h, D}(x)$ for all $x \in \bar{\Omega}$ and $t \geq 0$. Therefore, when estimating the coefficients $\gamma_{i, j}$, we may use the following estimates

$$
\frac{\left(A_{*}+\vartheta \phi\right)^{\alpha}}{\left(H_{*}+\vartheta \psi\right)^{\beta}} \leq\left\{\begin{array}{l}
\delta_{h}^{-\beta}\left(A_{*}+|\phi|\right)^{\alpha} \quad \text { if } \alpha>0, \\
\delta_{h}^{-\beta}\left(\min _{x \in \bar{\Omega}} \Sigma_{a, \varepsilon}(x)\right)^{\alpha} \quad \text { if } \alpha<0
\end{array}\right.
$$

as long as $\phi \geq-\phi_{*}$ and $\psi \geq-\psi_{*}$ and $0 \leq \vartheta \leq 1$.

Let $\theta_{*}$ be any constant such that

$$
1>\theta_{*}>\max \{1 / p, 1-r\} .
$$

Choose $m_{l}$ so that

$$
\begin{aligned}
& \max \left\{\frac{16 C_{12} C_{21} m_{a}^{p\left(r-1+\theta_{*}\right)}}{k_{1}^{(a)} k_{1}^{(h)}}, \frac{4 C_{12} m_{a}^{p\left(1-\theta_{*}\right)}}{k_{1}^{(a)}}, \frac{4 C_{21} m_{a}^{r}}{k_{1}^{(h)}}\right\} \leq \frac{1}{2} \\
& \quad \text { if } 0<m_{a} \leq m_{l} .
\end{aligned}
$$


Let $C_{\phi}$ and $C_{\psi}$ be positive numbers satisfying

$$
3\left(C_{\phi}+C_{\psi}\right) m_{a}^{\theta_{*} p} \leq m_{a} \quad \text { and } \quad 3\left(C_{\phi}+C_{\psi}\right) \leq \delta_{h} / 2 .
$$

Under these assumptions, we can easily prove that if

$$
\begin{aligned}
& -\phi_{*}(x) \leq \phi_{0}(x) \leq C_{\phi} m_{a}^{\theta_{*} p} \quad \text { and } \\
& -\psi_{*}(x) \leq \psi_{0}(x) \leq C_{\psi} \quad \text { for } x \in \bar{\Omega},
\end{aligned}
$$

then

$$
\begin{aligned}
& \sup _{x \in \Omega, 0<t<+\infty}\left|e^{\lambda t} \phi(x, t)\right| \leq 2\left(C_{\phi}+C_{\psi}\right) m_{a}^{\theta_{*} p} \quad \text { and } \\
& \sup _{x \in \Omega, 0<t<+\infty}\left|e^{\lambda t} \psi(x, t)\right| \leq 2\left(C_{\phi}+C_{\psi}\right) .
\end{aligned}
$$

This will be used in the next subsection.

\subsection{Collapse to almost decoupled patterns}

We here give a proof of Theorem 1.7, that is, we demonstrate the existence of solutions $(A(x, t), H(x, t))$ of $(1.1)-(1.3)$ which come to the neighborhood of $\left(A_{*}(x), H_{*}(x)\right)$ specified by Proposition 3.5.

If $H_{0}(x) \geq \max _{x \in \bar{\Omega}} \Sigma_{h, D}(x)$, then $\Psi(x, t)=H(x, t)-\Sigma_{h, D}(x)$ satisfies

$$
\left\{\begin{array}{l}
\tau \frac{\partial \Psi}{\partial t}=D \boldsymbol{\Lambda}_{h} \Psi-\mu_{h}(x) \Psi+\rho_{h}(A, H, x) \frac{A^{r}}{H^{s}} \text { for } x \in \Omega, t>0 \\
\boldsymbol{B}_{h} \Psi=0 \text { for } x \in \partial \Omega, t>0 \\
\Psi(x, 0)=H_{0}(x)-\Sigma_{h, D}(x) \text { for } x \in \Omega
\end{array}\right.
$$

Hence, the maximum principle implies $H(x, t) \geq \Sigma_{h, D}(x)$ for all $x \in \bar{\Omega}, t \geq 0$. In the following we assume $H(x, t) \geq \delta_{h}$.

Let $M(t)$ be a solution of the following initial value problem:

$$
\left\{\begin{array}{l}
\frac{d M}{d t}=-k_{1}^{(a)} M+\frac{C_{a}}{\delta_{h}^{q}} M^{p}+\max _{x \in \bar{\Omega}} \sigma_{a}(x), \\
M(0)=\max _{x \in \bar{\Omega}} A_{0}(x) .
\end{array}\right.
$$

Note that the algebraic equation (1.18) has two positive roots $\kappa_{a}$ and $K_{a}$ satisfying (1.19) and (1.20) when $m_{a}$ is sufficiently small. Therefore, if $0<$ $M(0)<K_{a}$, then the solution $M(t)$ of (3.64) is bounded for all $t>0$ and it approaches $\kappa_{a}$ as $t \rightarrow+\infty$. We observe from (1.19) that

$$
\kappa_{a}=m_{a}+O\left(m_{a}^{p}\right) \quad \text { as } m_{a} \downarrow 0 .
$$


Now, assuming $\max _{x \in \bar{\Omega}} A_{0}(x)<K_{a}$, we consider the following initialboundary value problem:

$$
\left\{\begin{array}{l}
\frac{\partial \bar{A}}{\partial t}=\varepsilon^{2} \boldsymbol{\Lambda}_{a} \bar{A}-\mu_{a}(x) \bar{A}+\frac{C_{a}}{\delta_{h}^{q}} \bar{A}^{p}+\sigma_{a}(x) \text { for } x \in \Omega, t>0, \\
\boldsymbol{B}_{a} \bar{A}=0 \text { for } x \in \partial \Omega, t>0 \\
\bar{A}(x, 0)=A_{0}(x) \text { for } x \in \Omega .
\end{array}\right.
$$

By the maximum principle, we see $M(t) \geq \bar{A}(x, t)$ for all $x \in \bar{\Omega}, t>0$. Since $M(t)$ converges to $\kappa_{a}$ as $t \rightarrow+\infty$, we obtain

$$
\limsup _{t \rightarrow+\infty} \max _{x \in \bar{\Omega}} \bar{A}(x, t) \leq \kappa_{a} .
$$

It is clear, by the maximum principle again, that the solution $A(x, t)$ of (1.1)-(1.3) satisfies

$$
A(x, t) \leq \bar{A}(x, t) \quad \text { for all } x \in \bar{\Omega}, t>0 .
$$

Therefore, it follows from (3.67) and (3.68) that $\max _{x \in \bar{\Omega}} A(x, t) \leq 2 \kappa_{a}$ whenever $t$ is sufficiently large, say $t \geq T$, and $m_{a}$ is sufficiently small.

To consider the asymptotic behavior of $(A(x, t), H(x, t))$, we need more precise estimate of $A(x, t)$ for $t$ sufficiently large. We see that $\bar{A}(x, t)$ converges to $\bar{A}_{*}(x)$ as $t \rightarrow+\infty$, where $\bar{A}_{*}(x)$ is a solution of

$$
\left\{\begin{array}{l}
\varepsilon^{2} \boldsymbol{\Lambda}_{a} \bar{A}_{*}-\mu_{a}(x) \bar{A}_{*}+\frac{C_{a}}{\delta_{h}^{q}} \bar{A}_{*}^{p}+\sigma_{a}(x)=0 \text { for } x \in \Omega, \\
\boldsymbol{B}_{a} \bar{A}_{*}=0 \text { for } x \in \partial \Omega .
\end{array}\right.
$$

Moreover, the convergence is uniform in $x \in \bar{\Omega}$. Indeed, it follows from (3.67) that $\max _{x \in \bar{\Omega}} \bar{A}(x, t) \leq 2 \kappa_{a}$ and $\max _{x \in \bar{\Omega}} \bar{A}_{*}(x) \leq 2 \kappa_{a}$ for $t>T$. Letting $\Phi(x, t)=\bar{A}(x, t+T)-\bar{A}_{*}(x)$, we see that $\Phi$ satisfies

$$
\left\{\begin{array}{l}
\Phi_{t}=\varepsilon^{2} \Lambda_{a} \Phi-\mu_{a}(x) \Phi+\frac{C_{a}}{\delta_{h}^{q}} p\left(\bar{A}_{*}+\theta \Phi\right)^{p-1} \Phi \text { for } x \in \Omega, t>0 \\
\boldsymbol{B}_{a} \Phi=0 \text { for } x \in \partial \Omega, t>0 \\
\Phi(x, 0)=A_{0}(x)-\bar{A}_{*}(x) \text { for } x \in \Omega
\end{array}\right.
$$

Since we can take $m_{a}$ so small that $-\mu_{a}(x)+p C_{a}\left(\bar{A}_{*}+\theta \Phi\right)^{p-1} / \delta_{h}^{q}<-2 \lambda_{0}<0$ where $\lambda_{0}$ is a positive constant, we obtain by the maximum principle that

$$
|\Phi(x, t)| \leq e^{-\lambda_{0} t} \max _{x \in \bar{\Omega}}\left|A_{0}(x)-\bar{A}_{*}(x)\right|,
$$

which implies $\lim _{t \rightarrow+\infty}\|\Phi(t)\|_{L^{\infty}}=0$. 
It follows from (3.68) and the arguments above that there exists a $T_{*}>0$ such that

$$
A(x, t) \leq \bar{A}_{*}(x)+m_{a}^{p} \quad \text { for all } x \in \bar{\Omega}, t>T_{*} .
$$

Since $\bar{A}_{*}(x)=O\left(m_{a}\right)$ as $m_{a} \downarrow 0$, we can apply the same argument as that used to prove the existence of the stationary solution $\left(A_{*}(x), H_{*}(x)\right)$, we obtain

$$
0 \leq \bar{A}_{*}(x)-\Sigma_{a, \varepsilon}(x) \leq \bar{\kappa} m_{a}^{p} \quad \text { for } x \in \bar{\Omega}
$$

whenever $m_{a}$ is sufficiently small, in which $\bar{\kappa}$ is an appropriate positive constant. Recall that $A_{*}(x)=\Sigma_{a, \varepsilon}(x)+\phi_{*}(x)$ with $\left\|\phi_{*}\right\|_{L^{\infty}} \leq \kappa m_{a}^{p}$. Hence, if we set $A\left(x, t+T_{*}\right)=A_{*}(x)+\phi_{a}(x, t)$, then $\phi_{a}$ must satisfy $\left\|\phi_{a}(t)\right\|_{\infty} \leq(\bar{\kappa}+1) m_{a}^{p}$. Therefore, the solution $A(x, t)$ satisfies

$$
\left|A(x, t)-A_{*}(x)\right| \leq(\kappa+\bar{\kappa}+1) m_{a}^{p}
$$

for $t$ sufficiently large.

Similarly, we can show

$$
\left|H(x, t)-H_{*}(x)\right| \leq\left(\kappa+\bar{\kappa}_{h}+1\right) m_{a}^{r},
$$

where $\bar{\kappa}_{h}$ is an appropriate positive constant. Indeed, by using the estimate $\max _{x \in \bar{\Omega}} A(x, t) \leq 2 m_{a}$ for $t>T_{*}$, we see

$$
H(x, t) \leq \bar{H}(x, t) \quad \text { for all } x \in \bar{\Omega}, t>T_{*},
$$

where $\bar{H}(x, t)$ is a solution of the following initial-boundary value problem:

$$
\left\{\begin{array}{l}
\frac{\partial \bar{H}}{\partial t}=D \boldsymbol{\Lambda}_{h} \bar{H}-\mu_{h}(x) \bar{H}+C_{h} \frac{\left(2 m_{a}\right)^{r}}{\delta_{h}^{s}}+\sigma_{h}(x) \text { for } x \in \Omega, t>0, \\
\boldsymbol{B}_{h} \bar{H}=0 \text { for } x \in \partial \Omega, t>0 \\
\bar{H}(x, 0)=H\left(x, T_{*}\right) \text { for } x \in \Omega .
\end{array}\right.
$$

We obtain that $\bar{H}(x, t)$ converges to $\bar{H}_{*}(x)$ as $t \rightarrow+\infty$ uniformly in $x \in \bar{\Omega}$. Here $\bar{H}_{*}(x)$ is a solution of

$$
\left\{\begin{array}{l}
D \boldsymbol{\Lambda}_{h} \bar{H}_{*}-\mu_{h}(x) \bar{H}_{*}+C_{h} \frac{\left(2 m_{a}\right)^{r}}{\delta_{h}^{s}}+\sigma_{h}(x)=0 \text { for } x \in \Omega, \\
\boldsymbol{B}_{h} \bar{H}_{*}=0 \text { for } x \in \partial \Omega .
\end{array}\right.
$$

It is easily seen that $\bar{H}_{*}=\Sigma_{h, D}(x)+O\left(m_{a}^{r}\right)$ as $m_{a} \downarrow 0$.

Consequently, it follows from (3.72) and (3.73) that there are positive constants $K_{\phi}$ and $K_{\psi}$ such that

$$
\left\|A(\cdot, t)-A_{*}\right\|_{L^{\infty}} \leq K_{\phi} m_{a}^{p}, \quad\left\|H(\cdot, t)-H_{*}\right\|_{L^{\infty}} \leq K_{\psi} m_{a}^{r} .
$$

Hence we can apply Proposition 3.5 to finish the proof of Theorem 1.7. 


\section{Concluding remarks}

In order to explain the meaning of the various conditions on the initial data leading to the collapse of patterns, it is convenient to introduce the following semi-bounded strips in the first quadrant of the $A H$-plane. First we define two functions $\Phi(A)$ and $\Psi(A)$ :

$\Phi(A)=\left[\frac{C_{a}(p-1)}{k_{1}^{(a)}(p-1)-q k_{2}^{(h)} / \tau} A^{p-1}\right]^{1 / q}, \quad \Psi(A)=\left[\left(\Gamma_{h} / \delta_{h}\right)^{k_{2}^{(h)} / k_{1}^{(h)}} \frac{C_{a}}{k_{1}^{(a)}} A^{p-1}\right]^{1 / q}$.

In defining $\Phi(A)$ we assume that $\tau>q k_{2}^{(h)} /\left[k_{1}^{(a)}(p-1)\right]$. For a positive number $A_{1}$, define semi-bounded strips $\mathscr{S}_{1}, \mathscr{S}_{2}$ and $\mathscr{S}_{3}$ by

$$
\begin{aligned}
\mathscr{S}_{1}\left(A_{1}\right) & =\left\{(A, H) \mid 0<A<A_{1}, H>\Phi\left(A_{1}\right)\right\}, \\
\mathscr{S}_{2}\left(A_{1}\right) & =\left\{(A, H) \mid 0<A<\min \left\{A_{1}, 1\right\}, H>\Psi\left(A_{1}\right)\right\}, \\
\mathscr{S}_{3} & =\left\{(A, H) \mid 0<A<K_{a}, H \geq \max _{x \in \bar{\Omega}} \Sigma_{h, D}(x)\right\} .
\end{aligned}
$$

Let $\mathscr{E}_{0}=\left\{\left(A_{0}(x), H_{0}(x)\right) \mid x \in \bar{\Omega}\right\}$. Then Condition (1.13) is equivalent to the condition $\mathscr{E}_{0} \subset \mathscr{S}_{1}\left(\max _{x \in \bar{\Omega}} A_{0}(x)\right)$, Condition (1.15) is equivalent to the condition $\mathscr{E}_{0} \subset \mathscr{S}_{2}\left(\max _{x \in \bar{\Omega}} A_{0}(x)\right)$ and Condition $(1.20)$ is equivalent to the condition $\mathscr{E}_{0} \subset \mathscr{S}_{3}$.

For a solution $(A(x, t), H(x, t))$ of the initial-boundary value problem (1.1)-(1.3), set

$$
\mathscr{E}(t)=\{(A(x, t), H(x, t)) \mid x \in \bar{\Omega}\} \quad \text { for } t \geq 0 .
$$

We may summarize our results as follows: If $\mathscr{E}\left(t_{0}\right)$ happens to be contained in one of the inhibitor-dominant strips $\mathscr{S}_{1}\left(A_{1}\right), \mathscr{S}_{2}\left(A_{1}\right)$, and $\mathscr{S}_{3}$ for some $A_{1}$ at certain time $t_{0}$, then the solution $(A(x, t), H(x, t))$ converges uniformly to $\left(A_{*}(x), H_{*}(x)\right)$ as $t \rightarrow+\infty$. Here we have defined $A_{*}(x) \equiv 0$ if $\sigma_{a}(x) \equiv 0$ and $H_{*}(x) \equiv 0$ if $\sigma_{h}(x) \equiv 0$. (Whenever $\sigma_{a} \equiv 0$, the almost decoupled pattern is in fact decoupled.)

The meaning of the conditions (1.13), (1.15) and (1.20) on initial data may be best illustrated in FIGURE 1 for the most frequently used case $(p, q, r, s)=$ $(2,1,2,0)$ :

$$
f(A, H)=-A+\frac{A^{2}}{H}+\sigma_{a}, \quad g(A, H)=-H+A^{2}+\sigma_{h},
$$

where $\sigma_{a}$ and $\sigma_{h}$ are nonnegative constants. In this case, $\Phi(A)=[1 /(1-1 / \tau)] A$ and $\Psi(A)=A$. 
a)

b)
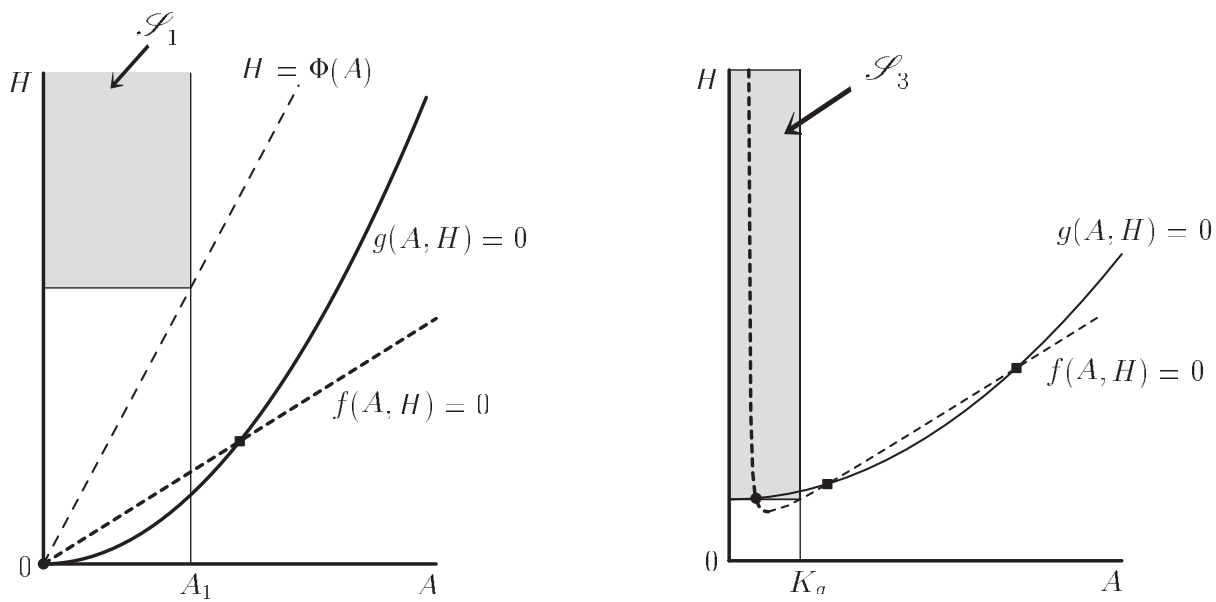

c1)
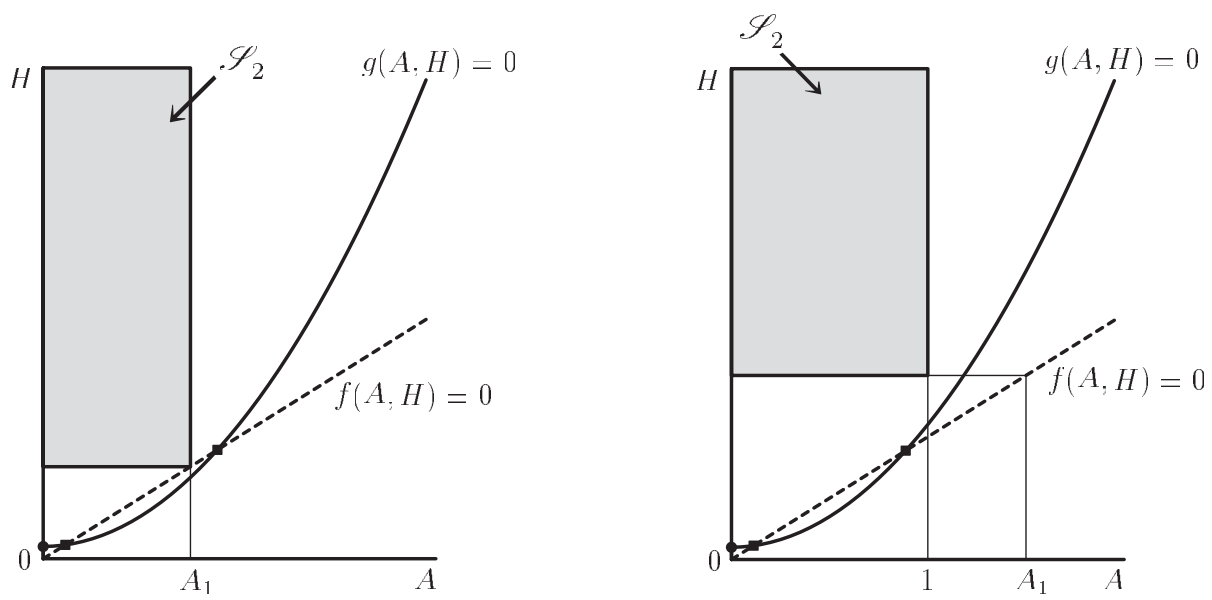

Fig. 1. The shaded rectangle in each figure shows an "inhibitor-dominant" strip. Fig. a) depicts Case I (Theorem 1.1), Fig. c1) and Fig. c2) display Case II (Theorem 1.2) and Fig. b) depicts Case III (Theorem 1.7). If $\sigma_{a}>0$ is sufficiently small compared with $\sigma_{h}$, then $f(A, H)=g(A, H)=0$ has three nonnegative solutions as in Fig. b), c1), c2). The smallest (in regard to $A$ ) is the almost decoupled pattern $\left(A_{*}, H_{*}\right)$. If $\sigma_{a}=\sigma_{h}=0$, then we set $\left(A_{*}, H_{*}\right)=(0,0)$. Once the set $\mathscr{E}(t)$ is contained in one of those strips at some moment $t$, the solution converges uniformly to $\left(A_{*}, H_{*}\right)$ as $t \rightarrow+\infty$. In the figures the filled circle stands for the point $\left(A_{*}, H_{*}\right)$; the filled square stands for a stationary solution. 
There are reaction-diffusion systems other than (GM) that admit the collapse of patterns. For example, the activator-inhibitor system with saturation, also proposed by Gierer and Meinhardt,

(SGM)

$$
\left\{\begin{array}{l}
a_{t}=D_{a} \Delta a-\mu a+\rho\left(c \frac{a^{p}}{h^{q}\left(1+\kappa a^{p}\right)}+\rho_{0}\right), \\
h_{t}=D_{h} \Delta h-v h+c^{\prime} \rho^{\prime} \frac{a^{r}}{h^{s}},
\end{array}\right.
$$

has the form (1.1) with $\rho_{a}=c \rho /\left(1+\kappa A^{p}\right)$. Here, $\kappa$ is a positive constant and (1.5) is satisfied uniformly on each compact subinterval of $0 \leq A<+\infty$, which is sufficient to prove all the statements. The following model proposed by MacWilliams [6] also has the same property:

$$
\left\{\begin{array}{l}
\frac{\partial u}{\partial t}=\varepsilon^{2} \Delta u-u+\frac{u^{p}}{u^{p}+v^{q}}+\sigma_{a} \\
\tau \frac{\partial v}{\partial t}=D \Delta v-v+\frac{\alpha u^{r}}{u^{r}+\beta}+\sigma_{h}
\end{array}\right.
$$

where $\varepsilon, D, \alpha, \beta, \tau$ are positive constants, and $\sigma_{a}, \sigma_{h}$ are nonnegative functions. The exponents $(p, q, r)$ satisfy (1.9) with $s=0$. MacWilliams used this system to simulate hydra transplantation experiments.

In view of Theorem 1.5, we can regard the collapse of patterns as a transient phenomenon en route to the almost decoupled pattern $\left(A_{*}, H_{*}\right)$ whenever the basic production term $\sigma_{a}$ for the activator is sufficiently small. In the case $\sigma_{a} \equiv \sigma_{h} \equiv 0$, due to the factors $v^{q}$ and $v^{r}$ in the denominator, $(u, v)=(0,0)$ is not an equilibrium of $(\mathrm{GM})$. Rather, it is a sort of singular rest point of saddle-point type, by which we mean that it is approachable only within a sector-like (or cusp-like) domain. The case $\sigma_{a} \equiv \sigma_{h} \equiv 0$ can be viewed as a limit of $\sigma_{a}(x) \rightarrow 0$ and $\sigma_{h}(x) \rightarrow 0$ subject to a certain condition on the size of $\left\|\sigma_{a}\right\|_{\infty}$ relative to $\left\|\sigma_{h}\right\|_{\infty}$. On the other hand, most reaction-diffusion systems do not have singularities in their nonlinearity. For example, the reaction terms of the FitzHugh-Nagumo system are polynomials; for a certain range of parameters it has a constant steady-state solution which is globally stable as an equilibrium of the ordinary differential equations, i.e., it models an excitable medium. In such a case patterns do collapse for a wide range of initial data. In particular, any solution is attracted to the constant steady-state solution once it enters a certain neighborhood. The almost decoupled pattern $\left(A_{*}, H_{*}\right)$ of the Gierer-Meinhardt system with $\sigma_{h}(x) \not \equiv 0$ shares the same property. In general, the presence of multiple stable equilibria or closed orbits makes the dynamics very complicated. It appears that some solutions of 
the initial-boundary value problem visit the neighborhood of several unstable equilibria or closed orbits one after another before completing a long journey to the final rest state.

It is equally important to know how the basic production terms affect the shape of stationary solutions. In this direction we obtained the following results in a separate paper [17], which were somewhat surprising to us. In the case of a homogeneous medium with $\sigma_{h}(x) \equiv 0$, there are several results on the existence of stationary solutions of (1.1)-(1.3) with boundary spikes (see, e.g., $[18,5,10,9,11,20,12])$. We consider the case where $\sigma_{a}(x)$ is a function of $x$ and see the influence of $\sigma_{a}(x)$ upon the shape of stationary solutions. In what follows we restrict ourselves to the simplest situation, that is to say, we consider the limiting system, called the shadow system, obtained by letting $D \rightarrow+\infty$ in spatial dimension one:

$$
\begin{aligned}
& \frac{\partial A}{\partial t}=\varepsilon^{2} \frac{\partial^{2} A}{\partial x^{2}}-A+\frac{A^{p}}{\xi^{q}}+\sigma_{a}(x) \quad \text { for } 0<x<l, t>0 \\
& \tau \frac{d \xi}{d t}=-\xi+\frac{1}{l \xi^{s}} \int_{0}^{l} A^{r} d x \quad \text { for } t>0 \\
& \frac{\partial A}{\partial x}(0, t)=\frac{\partial A}{\partial x}(l, t)=0 \quad \text { for } t>0 .
\end{aligned}
$$

We state our results after introducing a function which is crucial to describe the asymptotic form of the activator. Let $w(y)$ be the solution of the following boundary value problem:

$$
\left\{\begin{array}{l}
w^{\prime \prime}-w+w^{p}=0, \text { and } w>0 \text { for } 0<y<+\infty \\
w^{\prime}(0)=0, \quad \lim _{y \rightarrow+\infty} w(y)=0 .
\end{array}\right.
$$

It is well-known that the solution $w$ is unique and decays exponentially as $y \rightarrow+\infty: \sup _{0<y<\infty} e^{y} w(y)<+\infty$. Let $\Phi(y)$ be a solution of

$$
\left\{\begin{array}{l}
\Phi^{\prime \prime}-\Phi+p w^{p-1} \Phi+p w^{p-1}=0, \quad \text { for } 0<y<+\infty \\
\Phi^{\prime}(0)=0, \quad \lim _{y \rightarrow+\infty} \Phi(y)=0 .
\end{array}\right.
$$

This problem is known to have unique solutions (see, e.g., pp. 330-331 of [11]). The following theorem tells us how the term $\sigma_{a}(x)$ affects the shape of stationary solutions.

Theorem 4.1. Assume $\max _{0 \leq x \leq l} \sigma_{a}(x)>0$. Moreover, assume $\min _{0 \leq x \leq l} \sigma_{a}(x)>0$ if $0<r<1$. There exists an $\varepsilon_{0}>0$ such that for each 
$\varepsilon \in\left(0, \varepsilon_{0}\right)$ the shadow system has a pair of stationary solutions $\left(A_{1, \varepsilon}(x), \xi_{1, \varepsilon}\right)$ and $\left(A_{2, \varepsilon}(x), \xi_{2, \varepsilon}\right)$ satisfying

$$
\begin{aligned}
A_{1, \varepsilon}(x) & =\xi_{1, \varepsilon}^{q /(p-1)}\left\{w\left(\frac{x}{\varepsilon}\right)+o(1)\right\}+\sigma_{a}(x)+\sigma_{a}(0) \Phi\left(\frac{x}{\varepsilon}\right)+o(1) \\
\xi_{1, \varepsilon} & =\left\{\varepsilon\left(\frac{1}{l} \int_{0}^{\infty} w(z)^{r} d z+o(1)\right)\right\}^{-(p-1) /[q r-(p-1)(s+1)]} \\
A_{2, \varepsilon}(x) & =\xi_{2, \varepsilon}^{q /(p-1)}\left\{w\left(\frac{l-x}{\varepsilon}\right)+o(1)\right\}+\sigma_{a}(x)+\sigma_{a}(l) \Phi\left(\frac{l-x}{\varepsilon}\right)+o(1), \\
\xi_{2, \varepsilon} & =\left\{\varepsilon\left(\frac{1}{l} \int_{0}^{\infty} w(z)^{r} d z+o(1)\right)\right\}^{-(p-1) /[q r-(p-1)(s+1)]}
\end{aligned}
$$

as $\varepsilon \downarrow 0$. Here, the terms $o(1)$ in (4.6) and (4.8) are uniform in $x \in[0, l]$.

Rigorously speaking, we should notice that $\sigma_{a}(x)$ is not smooth in general, and hence the term $\sigma_{a}(x)$ in (4.6) and (4.8) should be replaced with $\Sigma_{\varepsilon}(x)$ which is the solution of (1.11). As to the stability of these stationary solutions, for example, we have

Theorem 4.2. Let $r=2$ and $1<p<5$. For each $\alpha \in\left(0, \alpha_{0}\right)$ where $\alpha_{0}$ is a sufficiently small number, one can choose an $\varepsilon_{1}>0$ so that for $0<\varepsilon<\varepsilon_{1}$, there exist $\tau_{1}>0$ and $\tau_{2}>0$ such that

(i) if $0<\tau<\tau_{1}$, then $\left(A_{1, \varepsilon}(x), \xi_{1, \varepsilon}\right)$ is asymptotically stable; and if $0<\tau<\tau_{2}$, then $\left(A_{2, \varepsilon}(x), \xi_{1, \varepsilon}\right)$ is asymptotically stable;

(ii) $\left(A_{1, \varepsilon}(x), \xi_{1, \varepsilon}\right)$ is unstable if $\tau>\tau_{1}$ and $\left(A_{2, \varepsilon}(x), \xi_{1, \varepsilon}\right)$ is unstable if $\tau>\tau_{2}$.

To sum up, under certain conditions on $r$ there exist stable steady-state solutions which have a very large spike at one of the end points of the interval, superimposed onto the distribution of the basic production term $\sigma_{a}(x)$. In these solutions, therefore, the boundary spike is the major part and the contribution of $\sigma_{a}(x)$ is relatively small as far as resulting patterns are concerned. This, however, does not rule out the possibility of the existence of a stable stationary solution with an interior spike.

The principal role of $\sigma_{a}$ seems to stabilize the system (i.e., to guarantee the boundedness of solutions) and avoid the collapse of patterns, without affecting very much the resulting patterns. This is an important observation from a biological view point. However, the situation changes drastically when we include the inhomogeneity in the cross-reaction coefficient $\rho_{a}(A, H, x)$. For example, Ren [13] discussed this problem. 


\section{References}

[1] Gierer, A. and Meinhardt, H., A theory of biological pattern formation, Kybernetik (Berlin), 12 (1972), 30-39.

[2] Jiang, H., Global existence of solutions of an activator-inhibitor system, Discrete Contin. Dyn. Syst., 14 (2006), 737-751.

[ 3 ] Koch, A. J. and Meinhardt, H., Biological pattern formation-from basic mechanisms to complex structures, Rev. Modern Physics, 66 (1994), 1481-1507.

[ 4 ] Li, M., Chen, S. and Qin, Y., Boundedness and blow up for the general activator-inhibitor model, Acta Math. Appl. Sinica (English Ser.), 11 (1995), 59-68.

[5] Lin, C.-S., Ni, W.-M. and Takagi, I., Large amplitude stationary solutions to a chemotaxis system, J. Differential Equations, 72 (1988), 1-27.

[6] MacWilliams, H. K., Numerical simulations of Hydra head regeneration using a proportionalregulating version of the Gierer-Meinhardt model, J. Theoret. Biol., 99 (1982), 681-703.

[ 7 ] Masuda, K. and Takahashi, K., Reaction-diffusion systems in the Gierer-Meinhardt theory of biological pattern formation, Japan J. Appl. Math., 4 (1987), 47-58.

[8 ] Ni, W.-M., Suzuki, K. and Takagi, I., The dynamics of a kinetic activator-inhibitor system, J. Differential Equations, 299 (2006), 426-465.

[9] Ni, W.-M. and Takagi, I., On the shape of least-energy solutions to a semilinear Neumann problem, Comm. Pure Appl. Math., 44 (1991), 819-851.

[10] Ni, W.-M. and Takagi, I., Locating the peaks of least-energy solutions to a semilinear Neumann problem, Duke Math. J., 70 (1993), 247-281.

[11] Ni, W.-M. and Takagi, I., Point condensation generated by a reaction-diffusion system in axially symmetric domains, Japan J. Indust. Appl. Math., 12 (1995), 327-365.

[12] Ni, W.-M., Takagi, I. and Yanagida, E., Stability analysis of point condensation solutions to a reaction-diffusion system, preprint.

[13] Ren, X., Least-energy solutions to a non-autonomous semilinear problem with small diffusion coefficient, Electron. J. Differential Equations, 1993 (1993), No. 05, 1-21.

[14] Rothe, F., Global Solutions of Reaction-Diffusion Systems, Lecture Notes in Mathematics, 1072, Springer, 1984.

[15] Suzuki, K. and Takagi, I., On the role of the source terms in an activator-inhibitor system proposed by Gierer and Meinhardt, Adv. Stud. Pure Math., 47-2, Math. Soc. Japan, Tokyo, 2007, 749-766.

[16] Suzuki, K. and Takagi, I., Behavior of solutions to an activator-inhibitor system with basic production terms, MI Lect. Note, 14, Kyushu Univ. Global COE Program, Fukuoka, 2009, 49-59.

[17] Suzuki, K. and Takagi, I., Collapse of patterns and effect of basic production terms in some reaction-diffusion systems, GAKUTO Internat. Ser. Math. Sci. Appl., 32, Gakkōtosho, Tokyo, 2010, 163-187.

[18] Takagi, I., Point-condensation for a reaction-diffusion system, J. Differential Equations, 61 (1986), 208-249.

[19] Turing, A. M., The chemical basis of morphogenesis, Philos. Trans. Roy. Soc. London, Ser. B, 237 (1952), 37-72.

[20] Wei, J., On the boundary spike layer solutions to a singularly perturbed neumann problem, J. Differential Equations, 134 (1997), 104-133.

[21] Wu, J. and Li, Y., Classical global solutions for the activator-inhibitor model, Acta Math. Appl. Sinica, 13 (1990), 501-505.

[22] Yanagihara, N., private communications. 


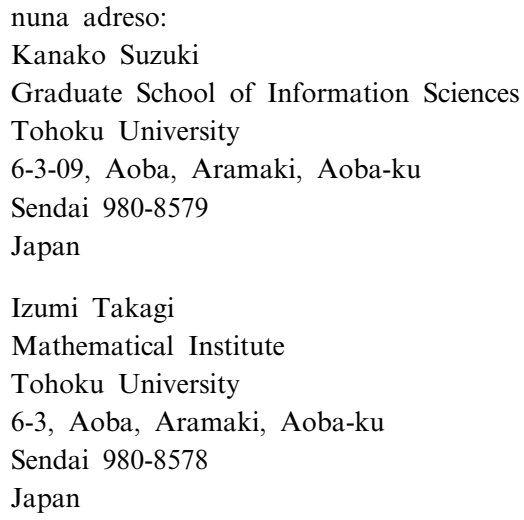

(Ricevita la 1-an de februaro, 2010)

(Reviziita la 7-an de junio, 2010) 